\title{
Newton representation of functions over natural integers having integral difference ratios
}

\author{
Patrick Cégielski*† Serge Grigorieff* ${ }^{* \ddagger} \quad$ Irène Guessarian*‡
}

October 15, 2018

\begin{abstract}
Different questions lead to the same class of functions from natural integers to integers: those which have integral difference ratios, i.e. verifying $f(a)-f(b) \equiv 0(\bmod (a-b))$ for all $a>b$.

We characterize this class of functions via their representations as Newton series. This class, which obviously contains all polynomials with integral coefficients, also contains unexpected functions, for instance all functions $x \mapsto\left\lfloor e^{1 / a} a^{x} x !\right\rfloor$, with $a \in \mathbb{Z} \backslash\{0,1\}$, and a function equal to Łe $x !\rfloor$ except on 0 . Finally, to study the complement class, we look at functions $\mathbb{N} \rightarrow \mathbb{R}$ which are not uniformly close to any function having integral difference ratios.
\end{abstract}

Keywords.

\section{Contents}

1 Introduction

1.1 What is in the paper?

1.2 Where does the problem come from?

1.2.1 Closure properties of lattices of subsets of $\mathbb{N}$

1.2.2 Uniform continuity properties related to varieties of groups

2 Characterization of the integral difference ratios property

2.1 Newton series of functions $\mathbb{N} \rightarrow \mathbb{Z}$ and integral difference ratios

2.2 Preparatory lemmas for the proof of Theorem 2.5

2.3 Proof of Theorem 2.5

3 Examples of functions having integral difference ratios

3.1 Main examples: around the factorial function

3.2 Algebra of functions having integral difference ratios and applications

3.3 Examples with generalized hyperbolic functions

3.4 Asymptotic equivalence

4 Outside the family of functions with integral difference ratios

4.1 Uniform closeness

4.2 A general negative result for uniform closeness to functions having integral difference ratios

4.3 Non integral polynomial functions

4.4 Functions around the exponential functions

4.5 Functions around the factorial function

*Partially supported by TARMAC ANR agreement 12 BS02 00701.

${ }^{\dagger}$ LACL, EA 4219, Université Paris-Est Créteil, France, email : cegielski@u-pec.fr.

${ }^{\ddagger}$ LIAFA, CNRS and Université Paris-Diderot, France, firstname.name@liafa.univ-parisdiderot.fr. 


\section{Introduction}

\subsection{What is in the paper?}

We consider the following question: what are the functions $f: \mathbb{N} \rightarrow \mathbb{Z}$ having integral difference ratios, i.e. such that $a-b$ always divides $f(a)-f(b)$ ?

Our motivation for such functions came from questions in theoretical computer science, cf. \$1.2, But these functions are clearly interesting per se.

In $\$ 2$ we characterize functions having integral difference ratios as the $\mathbb{N} \rightarrow \mathbb{Z}$ functions associated to Newton series such that the least common multiple of $2,3, \ldots, k$ divides the $k$-th coefficient.

$\$ 3$ is devoted to examples of functions having integral difference ratios. Polynomials with coefficients in $\mathbb{Z}$ are trivial examples. The above characterization shows that there are a lot of non polynomial examples. It turns out that some of them are simply expressible. For instance (cf. \$3.1), the functions

$$
x \mapsto\left\lfloor e^{1 / a} a^{x} x !\right\rfloor \quad \text { with } a \in \mathbb{Z} \backslash\{0,1\} \quad, \quad x \mapsto \begin{cases}1 & \text { if } \quad x=0 \\ \lfloor e x !\rfloor & \text { if } \quad x \in \mathbb{N} \backslash\{0\}\end{cases}
$$

There are also examples of such functions which oscillate in a periodic way between several simple expressions of the values, for instance (cf. \$3.3) the functions which map $x \in \mathbb{N}$ to

$$
\left\{\begin{array} { l l l } 
{ \lfloor \operatorname { c o s h } ( 1 / 2 ) 2 ^ { x } x ! \rfloor } & { \text { if } } & { x \in 2 \mathbb { N } } \\
{ \lfloor \operatorname { s i n h } ( 1 / 2 ) 2 ^ { x } x ! \rfloor } & { \text { if } } & { x \in 2 \mathbb { N } + 1 }
\end{array} \quad \left\{\begin{array}{lll}
0 & \text { if } & x=0 \\
\left\lfloor\cosh (1 / 2) 2^{x} x !\right\rfloor & \text { if } & x \in 2 \mathbb{N}+1 \\
\left\lfloor\sinh (1 / 2) 2^{x} x !\right\rfloor & \text { if } & x \in 2 \mathbb{N}+2
\end{array}\right.\right.
$$

To witness the richness of the family of functions having integral difference ratios, we prove (cf. \$3.4) that this family contains functions asymptotically equivalent to large enough functions (larger than $(2 e+\varepsilon)^{x}$ for some $\varepsilon>0$ suffices).

Finally, in 4 we show that the above examples are kind of exceptions: as can be expected, most functions similar to the above examples do not have rational difference ratios. Worse, they are not uniformly close to any function having rational difference ratios. In fact, it turns out that proving non uniform closeness is a very manageable tool to prove failure of the integral difference ratios property. First, we use (cf. 4.2 ) a classical result from the theory of uniform distribution modulo one to get a general result about non uniform closeness: if $\inf \left\{\left|\lambda_{x}-\lambda_{y}\right| \mid x, y \in \mathbb{N}, x \neq y\right\}>0$ then, for almost all real number $\alpha$ (in the sense of Lebesgue measure), the function $x \mapsto \alpha \lambda_{x}$ is uniformly close to no function having integral difference ratios. Then we look at simple particular classes of functions.

- For non constant polynomials with real coefficients, we show (cf. 84.3) that closeness to a function having integral difference ratios holds if and only if all coefficients are in $\mathbb{Z}$ (in which case this polynomial function has integral difference ratios).

- For $\alpha \neq 0$, all exponential functions $\alpha k^{x}$ (with $k \in \mathbb{N} \backslash\{0,1\}$ ) fail to be uniformly close to a function having integral difference ratios (cf. 4.4).

- As seen by the examples mentioned supra, the case of functions $\alpha a^{x} x$ ! is more delicate. We study it in 4.5 . 


\subsection{Where does the problem come from?}

A function $f: \mathbb{N} \rightarrow \mathbb{Z}$ is said to have integral difference ratios if $\frac{f(a)-f(b)}{a-b} \in \mathbb{Z}$ for all $b<a$. As far as we know, the class of functions $\mathbb{N} \rightarrow \mathbb{Z}$ with integral difference ratios emerged in Pin \& Silva, 2011 [18 (see also $\$ 4.2$ in 17]) and in our paper [2]. In the latter, we showed that the integral difference ratio property characterizes closure of lattices of regular subsets of $\mathbb{N}$ under inverse image by $f$ (Theorem 1.1 below).

\subsubsection{Closure properties of lattices of subsets of $\mathbb{N}$.}

Theorem $1.1([2])$. Let $S u c: \mathbb{N} \rightarrow \mathbb{N}$ be the successor function and let $f: \mathbb{N} \rightarrow$ $\mathbb{N}$. The following conditions are equivalent.

(i) The map $f$ is non decreasing and satisfies $f(a) \geq a$ and has integral difference ratios.

(ii) For every finite set $L \subset \mathbb{N}$, the smallest lattice of subsets of $\mathbb{N}$ containing $L$ and closed under $S u c^{-1}$ is also closed under $f^{-1}$.

(iii) For every arithmetic progression $L=q+r \mathbb{N}, q, r \in \mathbb{N}, r>0$, the smallest lattice of subsets of $\mathbb{N}$ containing $L$ and closed under $S u c^{-1}$ is also closed under $f^{-1}$.

(iv) Every lattice of regular subsets of $\mathbb{N}$ which is closed under Suc ${ }^{-1}$ is closed under $f^{-1}$.

\subsubsection{Uniform continuity properties related to varieties of groups.}

To state the result in 18, we need to recall some of the involved basic notions though they are not used anywhere else in the paper.

Definition 1.2. 1. A class of finite monoids is a variety if it closed under taking submonoids, quotients and finite direct products.

2. Given a variety $\mathbf{V}$ of finite monoids and a monoid $M$, the pseudo-metric $d_{\mathbf{V}}: M \times M \rightarrow[0,1]$ is defined by $d_{\mathbf{V}}(x, y)=2^{-k}$ where $k$ is least such that $\varphi(x) \neq \varphi(y)$ for some morphism $\varphi: M \rightarrow F$ such that $F \in \mathbf{V}$ has $k$ elements (and $k=+\infty$ if there is no such morphism).

Theorem 1.3 ([18],cf. also $\S 4.2$ in [17])). Let $\mathbf{G}$ be the variety of finite groups. Consider the monoid $(\mathbb{Z},+)$ and let $f: \mathbb{Z} \rightarrow \mathbb{Z}$. The following conditions are equivalent.

(i) $f$ is $d_{\mathbf{V}}$-uniformly continuous for every subvariety $\mathbf{V}$ of $\mathbf{G}$.

(ii) $u-v$ divides $f(u)-f(v)$ for all $u, v \in \mathbb{Z}$.

\section{Characterization of the integral difference ra- tios property}

To get a characterization of functions having integral difference ratios, we use Newton series [15, 1], originally introduced to study functions from $\mathbb{R}$ to $\mathbb{R}$, but here reduced to functions from $\mathbb{N}$ to $\mathbb{Z}$. 


\subsection{Newton series of functions $\mathbb{N} \rightarrow \mathbb{Z}$ and integral differ- ence ratios}

Our first result, Theorem 2.5, involves notions recalled in Definition 2.3 and Proposition 2.2 below.

Definition 2.1 (Newton representation for functions $\mathbb{N} \rightarrow \mathbb{Z}$ ). A map $f: \mathbb{N} \rightarrow$ $\mathbb{Z}$ has a Newton representation if there exists some sequence $\left(a_{k}\right)_{k \in \mathbb{N}}$ such that, for all $x \in \mathbb{N}$, the value $f(x)$ is equal to the sum of the series

$$
f(x)=\sum_{k \in \mathbb{N}} a_{k} \frac{\prod_{i=0}^{k-1}(x-i)}{k !}=\sum_{k \in \mathbb{N}} a_{k}\left(\begin{array}{l}
x \\
k
\end{array}\right)
$$

Proposition 2.2 (Newton series correspondence). A bijective correspondence between sequences $\left(a_{k}\right)_{k \in \mathbb{N}}$ of integers in $\mathbb{Z}$ and maps $f: \mathbb{N} \rightarrow \mathbb{Z}$ is set up by the Newton representation (11) where, for $k \in \mathbb{N}$,

$$
a_{k}=\sum_{i=0}^{i=k}(-1)^{k-i} f(i)\left(\begin{array}{l}
k \\
i
\end{array}\right)
$$

Proof. Observe that, for every $x \in \mathbb{N}$, the binomial coefficient $\left(\begin{array}{l}x \\ k\end{array}\right)$ is null for $k>x$, hence the infinite series defining $f(x)$ in (1) reduces to a finite sum for any given non negative $x$. This removes any convergence problem. Since the binomial coefficients are in $\mathbb{N}$, for every sequence $\left(a_{k}\right)_{k \in \mathbb{N}} \in \mathbb{Z}^{\mathbb{N}}$, equation (1) represents a map from $\mathbb{N}$ into $\mathbb{Z}$.

Conversely, every $f: \mathbb{N} \rightarrow \mathbb{Z}$ has a unique such representation since (1) insures that

$$
a_{0}=f(0) \quad, \quad a_{k+1}=f(k+1)-\sum_{i=0}^{i=k} a_{i}\left(\begin{array}{c}
k+1 \\
i
\end{array}\right) \in \mathbb{Z} .
$$

Inverting the binomial lower triangular matrix $B_{k}=\left(\left(\begin{array}{l}i \\ j\end{array}\right)\right)_{0 \leq i, j \leq k}$ in equality

$$
\left(\begin{array}{c}
f(0) \\
f(1) \\
f(2) \\
f(3) \\
\vdots \\
f(k)
\end{array}\right)=\left(\begin{array}{cccccc}
1 & & & & & \\
1 & 1 & & & & \\
1 & 2 & 1 & & & \\
1 & 3 & 3 & 1 & & \\
\vdots & \vdots & \vdots & \ldots & \vdots \\
1 & \left(\begin{array}{c}
k \\
1
\end{array}\right) & \left(\begin{array}{c}
k \\
2
\end{array}\right) & \cdots & \left(\begin{array}{c}
k \\
k-1
\end{array}\right) & 1
\end{array}\right)\left(\begin{array}{c}
a_{0} \\
a_{1} \\
a_{2} \\
a_{3} \\
\vdots \\
a_{k}
\end{array}\right)
$$

we get formula (2) since the inverse of $B_{k}$ is the triangular matrix $\widetilde{B}_{k}=$ $\left((-1)^{i-j}\left(\begin{array}{l}i \\ j\end{array}\right)\right)_{0 \leq i, j \leq k}$ (cf. for instance [26]). Indeed, the $(i, j)$ element of $B_{k} \widetilde{B}_{k}$ is $\sum_{\ell=0}^{\ell=k}\left(\begin{array}{l}i \\ \ell\end{array}\right)(-1)^{\ell-j}\left(\begin{array}{l}\ell \\ j\end{array}\right)$. Recall that (cf [7] page 174) $\left(\begin{array}{l}i \\ \ell\end{array}\right)\left(\begin{array}{l}\ell \\ j\end{array}\right)=\left(\begin{array}{l}i \\ j\end{array}\right)\left(\begin{array}{l}i-j \\ \ell-j\end{array}\right)$. Hence,

$\sum_{\ell=0}^{\ell=k}\left(\begin{array}{l}i \\ \ell\end{array}\right)(-1)^{\ell-j}\left(\begin{array}{l}\ell \\ j\end{array}\right)=\left(\begin{array}{l}i \\ j\end{array}\right) \sum_{\ell=0}^{\ell=k}(-1)^{\ell-j}\left(\begin{array}{c}i-j \\ \ell-j\end{array}\right)=\left(\begin{array}{l}i \\ j\end{array}\right)(1-1)^{i-j}= \begin{cases}0 & \text { if } i \neq j \\ 1 & \text { if } i=j\end{cases}$ 
which proves that $\widetilde{B}_{k}$ is the inverse of $B_{k}$.

To state the main Theorem of the present section, we need to recall another classical notion.

Definition 2.3. For $k \in \mathbb{N}, k \geq 1$, lcm $(k)$ is the least common multiple of all positive integers less than or equal to $k$. By convention, $\operatorname{lcm}(0)=1$.

Remark 2.4. The Neperian logarithm of the $l \mathrm{~cm}$ function was introduced by Chebychev, 1852 [4: letting $\ell(p, x)=\left\lfloor\log _{p}(x)\right\rfloor$ be the greatest integer $k$ such that $p^{k} \leq x$,

$$
\psi(x)=\sum\{\ell(p, x) \log p \mid p \leq x, p \text { prime }\}=\log (\operatorname{lcm}(x)) .
$$

A variant of the prime number theorem insures that the Chebychev function $\psi(x)$ is asymptotically equivalent to $x$, i.e. $\lim _{n \rightarrow+\infty} \psi(x) / x=1$. Thus, for any $\varepsilon>0$, we have $\log (\operatorname{lcm}(x))=x(1+o(x))$ hence $\operatorname{lcm}(x)=e^{x(1+o(x))}=$ $\left(e^{1+o(x)}\right)^{x}=(e+o(x))^{x}$, i.e. for every $\varepsilon>0$, for all $x$ large enough,

$$
(e-\varepsilon)^{x} \leq l c m(x) \leq(e+\varepsilon)^{x} .
$$

Simple lower and upper bounds of $l \mathrm{~cm}$ are known: $2^{n} \leq l \mathrm{~cm}(n)$ for $n \geq 7$ (cf. formula (9) in 14 for $n \geq 9$ plus direct check for $n=7,8)$ and $l \mathrm{~cm}(n)<3^{n}$ for all $n \in \mathbb{N}$ (cf. [8]).

It is known (22]) that $\psi(x)$ (resp. lcm $(x))$ oscillates around $x$ (resp. $\left.e^{x}\right)$ : for some $K>0$, there are infinitely many $x$ 's such that $l$ cm $(x)<e^{x-K \sqrt{x}}$ and infinitely many $x$ 's such that $\operatorname{lcm}(x)>e^{x+K \sqrt{x}}$. Nevertheless, the relative oscillation is in $o\left(e^{x}\right)$ (20] Theorem 8): letting $a=2 \times 10^{7}$, for all $x \geq 2$,

$$
e^{x-a\left(x / \log ^{4} x\right)}<\operatorname{lcm}(x)<e^{x+a\left(x / \log ^{4} x\right)} .
$$

Assuming Riemann's hypothesis, a better approximation is possible (23] Theorem 10):

Riemann's hypothesis proves $e^{x-\left(\sqrt{x} \log ^{2}(x) / 8 \pi\right)}<\operatorname{lcm}(x)<e^{x+\left(\sqrt{x} \log ^{2}(x) / 8 \pi\right)}$.

For recent results around the $l \mathrm{~cm}$ function, see [19, 9, 5, 6, 3.

Theorem 2.5. Let $f: \mathbb{N} \rightarrow \mathbb{Z}$ be a function with Newton representation $\sum_{k \in \mathbb{N}} a_{k}\left(\begin{array}{l}x \\ k\end{array}\right)$. The following conditions are equivalent:

(i) $f$ has integral difference ratios, i.e. $\frac{f(a)-f(b)}{a-b} \in \mathbb{Z}$ for all $b \neq a$.

(ii) $l c m(k)$ divides $a_{k}$ for all $k \in \mathbb{N}$.

Proof. See $2.2,2.3 .1,2.3 .2$

We now state a corollary whose proof does not need the machinery of the proof of Theorem 2.5

Corollary 2.6. If $k$ ! divides $a_{k}$ for all $k \in \mathbb{N}$ then $f$ has integral difference ratios.

Proof. Let $a_{k}=k ! b_{k}$ (with $\left.b_{k} \in \mathbb{Z}\right)$; then $f(x)=\sum_{k \in \mathbb{N}} a_{k}\left(\begin{array}{l}x \\ k\end{array}\right)=b_{0}+b_{1} x+$ $b_{2} x(x-1)+b_{3} x(x-1)(x-2)+\cdots$. For $a, b \in \mathbb{N}$ and $N>\max (a, b), f(a)-f(b)$ is the difference of the values on $a, b$ of the polynomial $\sum_{k<N} b_{k} \prod_{i=0}^{i=k-1}(x-i)$ which has coefficients in $\mathbb{Z}$, hence $a-b$ divides $f(a)-f(b)$. 


\subsection{Preparatory lemmas for the proof of Theorem 2.5}

The proof of Theorem 2.5 relies on three lemmas whose proofs are elementary.

Lemma 2.7. If $0 \leq n-k<p \leq n$ then $p$ divides $\operatorname{lcm}(k)\left(\begin{array}{l}n \\ k\end{array}\right)$.

Proof. By induction on $n \geq 1$. The initial case $n=1$ is trivial since condition $0 \leq n-k<p \leq n$ yields $p=k=1$. Induction step: assuming the result for $n$, we prove it for $n+1$. Suppose $0 \leq n+1-k<p \leq n+1$.

First case: $p \leq n$. Then we have $0 \leq n-k<p \leq n$ and $0 \leq n-(k-1)<p \leq n$, so that, by induction hypothesis, $p$ divides $\operatorname{lcm}(k)\left(\begin{array}{l}n \\ k\end{array}\right)$ and $p$ divides $\operatorname{lcm}(k-$ 1) $\left(\begin{array}{c}n \\ k-1\end{array}\right)$. A fortiori, $p$ divides $\operatorname{lcm}(k)\left(\begin{array}{l}n \\ k\end{array}\right)+\operatorname{lcm}(k)\left(\begin{array}{c}n \\ k-1\end{array}\right)=\operatorname{lcm}(k)\left(\begin{array}{c}n+1 \\ k\end{array}\right)$ (by Pascal's formula).

Second case: $p=n+1$. Then $k \geq 1$ and $\operatorname{lcm}(k)\left(\begin{array}{c}n+1 \\ k\end{array}\right)=(n+1) \frac{l c m(k)}{k}\left(\begin{array}{c}n \\ k-1\end{array}\right)$ hence $p=n+1$ divides $\operatorname{lcm}(k)\left(\begin{array}{c}n+1 \\ k\end{array}\right)$.

Lemma 2.8. If $n, k, b \in \mathbb{N}$ and $k \leq b$ then $n$ divides $A_{k, b}^{n}=\operatorname{lcm}(k)\left(\left(\begin{array}{c}b+n \\ k\end{array}\right)-\left(\begin{array}{l}b \\ k\end{array}\right)\right)$.

Proof. We argue by double induction on $k$ and $b$ with the conditions

$\left(\mathcal{P}_{k, b}\right) \quad \forall n \in \mathbb{N}, \quad n$ divides $A_{k, b}^{n} \quad, \quad\left(\mathcal{P}_{k}\right) \quad \forall b \geq k, \forall n \in \mathbb{N}, n$ divides $A_{k, b}^{n}$.

Conditions $\left(\mathcal{P}_{0}\right)$ and $\left(\mathcal{P}_{1}\right)$ are trivial since $A_{0, b}^{n}=0$ and $A_{1, b}^{n}=n$.

Suppose $k \geq 1$ and $\left(\mathcal{P}_{k}\right)$ is true. To prove $\left(\mathcal{P}_{k+1}\right)$, we prove by induction on $b \geq k+1$ that $\left(\mathcal{P}_{k+1, b}\right)$ holds.

In the basic case $b=k+1$, we have

$$
\begin{aligned}
A_{k+1, k+1}^{n} & =\operatorname{lcm}(k+1)\left(\left(\begin{array}{c}
k+1+n \\
k+1
\end{array}\right)-\left(\begin{array}{c}
k+1 \\
k+1
\end{array}\right)\right) \\
& =l c m(k+1)\left(\left(\begin{array}{c}
k+n \\
k
\end{array}\right)+\left(\begin{array}{c}
k+n \\
k+1
\end{array}\right)-1\right) \quad \text { by Pascal's relation } \\
& =\operatorname{lcm}(k+1)\left(\left(\begin{array}{c}
k+n \\
k
\end{array}\right)-\left(\begin{array}{l}
k \\
k
\end{array}\right)\right)+l c m(k+1)\left(\begin{array}{c}
k+n \\
k+1
\end{array}\right) \\
& =\frac{\operatorname{lcm}(k+1)}{\operatorname{lcm}(k)} A_{k, k}^{n}+\operatorname{lcm}(k+1)\left(\begin{array}{c}
k+n \\
k+1
\end{array}\right)
\end{aligned}
$$

Since $\left(\mathcal{P}_{k, k}\right)$ holds (induction hypothesis on $k$ ), $n$ divides $A_{k, k}^{n}$ hence divides the first term. If $n \leq k+1$ then $n$ divides $l c m(k+1)$ hence divides the second term. If $n>k+1$, applying Lemma 2.7 with $n^{\prime}=k+n, p^{\prime}=n$ and $k^{\prime}=k+1$, shows that $n=p^{\prime}$ divides the second term. Thus, $n$ divides $A_{k+1, k+1}^{n}$ and $\left(\mathcal{P}_{k+1, k+1}\right)$ holds.

Suppose now that $\left(\mathcal{P}_{k+1, c}\right)$ holds for $k+1 \leq c \leq b$. We prove $\left(\mathcal{P}_{k+1, b+1}\right)$. 
Using Pascal's relation, we get

$$
\begin{aligned}
A_{k+1, b+1}^{n} & =\operatorname{lcm}(k+1)\left(\left(\begin{array}{c}
b+1+n \\
k+1
\end{array}\right)-\left(\begin{array}{l}
b+1 \\
k+1
\end{array}\right)\right) \\
& =\operatorname{lcm}(k+1)\left(\left(\begin{array}{c}
b+n \\
k
\end{array}\right)+\left(\begin{array}{l}
b+n \\
k+1
\end{array}\right)-\left(\begin{array}{l}
b \\
k
\end{array}\right)-\left(\begin{array}{c}
b \\
k+1
\end{array}\right)\right) \\
& \left.=l c m(k+1)\left(\left(\begin{array}{c}
b+n \\
k
\end{array}\right)-\left(\begin{array}{l}
b \\
k
\end{array}\right)\right)+\left(\left(\begin{array}{l}
b+n \\
k+1
\end{array}\right)-\left(\begin{array}{c}
b \\
k+1
\end{array}\right)\right)\right) \\
& =\left(\frac{l c m(k+1)}{l c m(k)} A_{k, b}^{n}\right)+A_{k+1, b}^{n}
\end{aligned}
$$

Since $\left(\mathcal{P}_{k, b}\right)$ and $\left(\mathcal{P}_{k+1, b}\right)$ hold, $n$ divides both terms of the above sum hence $n$ divides $A_{k+1, b+1}^{n}$ and $\left(\mathcal{P}_{k+1, b+1}\right)$ holds.

The following is an immediate consequence of Lemma 2.8 (set $a=b+n)$.

Lemma 2.9. If $a \geq b$ then $a-b$ divides $\operatorname{lcm}(k)\left(\left(\begin{array}{l}a \\ k\end{array}\right)-\left(\begin{array}{l}b \\ k\end{array}\right)\right)$ for all $k \leq b$.

\subsection{Proof of Theorem 2.5}

\subsubsection{Proof of (i) $\Rightarrow$ (ii)}

We suppose that $f(x)=\sum_{k \in \mathbb{N}} a_{k}\left(\begin{array}{l}x \\ k\end{array}\right)$ has integral difference ratios and we show that $\operatorname{lcm}(k)$ divides $a_{k}$ for all $k \in \mathbb{N}$.

Claim 1. For all $k \geq 1, k$ divides $a_{k}$.

The proof is by induction. Recall $f(k)=\sum_{i=0}^{k}\left(\begin{array}{l}k \\ i\end{array}\right) a_{i}$.

Induction Basis: The case $k=1$ is trivial. For $k=2$, observe that 2 divides $f(2)-f(0)=2 a_{1}+a_{2}$ hence 2 divides $a_{2}$.

Inductive Step: assuming that $\ell$ divides $a_{\ell}$ for every $\ell \leq k$, we prove that $k+1$ divides $a_{k+1}$. Observe that

$$
\begin{aligned}
f(k+1)-f(0) & =(k+1) a_{1}+\left(\sum_{i=2}^{k}\left(\begin{array}{c}
k+1 \\
i
\end{array}\right) a_{i}\right)+a_{k+1} \\
& =(k+1) a_{1}+\left(\sum_{i=2}^{k}(k+1) \frac{a_{i}}{i}\left(\begin{array}{c}
k \\
i-1
\end{array}\right)\right)+a_{k+1}
\end{aligned}
$$

By the induction hypothesis, $\frac{a_{i}}{i}$ is an integer for $i \leq k$. Since $f$ has integral difference ratios, $k+1$ divides $f(k+1)-f(0)$ hence $k+1$ divides the last term $a_{k+1}$ of the sum.

Claim 2. For all $1 \leq p \leq k, p$ divides $a_{k}$. Hence, lcm $(k)$ divides $a_{k}$.

The case $p=1$ is trivial. We use induction on $p \geq 2$.

- Basic case: 2 divides $a_{k}$ for all $k \geq 2$. We argue by induction on $k \geq 2$. Claim 1 gives the base case: 2 divides $a_{2}$. Induction step: assuming that 2 divides $a_{i}$ for all $2 \leq i \leq k$ we prove that 2 divides $a_{k+1}$. Two cases can occur. Subcase $1: k+1$ is odd. Then 2 divides $f(k+1)-f(1)$. Now,

$$
f(k+1)-f(1)=k a_{1}+\left(\sum_{i=2}^{k} a_{i}\left(\begin{array}{c}
k+1 \\
i
\end{array}\right)\right)+a_{k+1},
$$


$k$ is even and 2 divides the $a_{i}$ for $2 \leq i \leq k$ by the induction hypothesis, hence, 2 divides $a_{k+1}$.

Subcase 2: $k+1$ is even. Then 2 divides $f(k+1)-f(0)$. Now,

$$
f(k+1)-f(0)=(k+1) a_{1}+\left(\sum_{i=2}^{k} a_{i}\left(\begin{array}{c}
k+1 \\
i
\end{array}\right)\right)+a_{k+1},
$$

$k+1$ is even and 2 divides the $a_{i}$ for $2 \leq i \leq k$ by the induction hypothesis, thus, 2 divides $a_{k+1}$.

- Induction step: assuming that $p \geq 2$ and, for all $q \leq p, q$ divides $a_{\ell}$ for all $\ell \geq q$, we prove that $p+1$ divides $a_{k}$ for all $k \geq p+1$.

We use induction on $k \geq p+1$. Claim 1 gives the base case: $p+1$ divides $a_{p+1}$. Induction step: assuming that $p+1$ divides $a_{i}$ for all $p+1 \leq i \leq k$ we prove that $p+1$ divides $a_{k+1}$. Since $f$ has integral difference ratios, $p+1$ divides $f(k+1)-f(k-p)$ which is equal to

$$
\sum_{i=1}^{i=k-p} a_{i}\left(\left(\begin{array}{c}
k+1 \\
i
\end{array}\right)-\left(\begin{array}{c}
k-p \\
i
\end{array}\right)\right)+\left(\sum_{i=k+1-p}^{i=k} a_{i}\left(\begin{array}{c}
k+1 \\
i
\end{array}\right)\right)+a_{k+1}
$$

Let us first look at the terms of the first sum corresponding to $1 \leq i \leq p$. The induction hypothesis (on $p$ ) insures that $q$ divides $a_{k}$ for all $q \leq p$ and $k \geq q$. In particular, (letting $k=i) l c m(i)$ divides $a_{i}$. Since $(k+1)-(k-p)=p+1$, Lemma 2.8 insures that $p+1$ divides $l c m(i)\left(\left(\begin{array}{c}k+1 \\ i\end{array}\right)-\left(\begin{array}{c}k-p \\ i\end{array}\right)\right)$. A fortiori, $p+1$ divides $a_{i}\left(\left(\begin{array}{c}k+1 \\ i\end{array}\right)-\left(\begin{array}{c}k-p \\ i\end{array}\right)\right)$.

We now turn to the terms of the first sum corresponding to $p+1 \leq i \leq k-p$ (if there are any). The induction hypothesis (on $k$ ) insures that $p+1$ divides $a_{i}$ for all $p+1 \leq i \leq k$

Thus, each term of the first sum is divisible by $p+1$.

Consider now the terms of the second sum. By the induction hypothesis (on $k$ ), $p+1$ divides $a_{i}$ for all $p+1 \leq i \leq k$. It remains to look at the terms associated with the $i$ 's such that $k+1-p \leq i \leq p$ (there are such $i$ 's in case $k+1-p<p+1)$. For such $i$ 's we have $0 \leq(k+1)-i \leq(k+1)-p<p+1 \leq k+1$ and Lemma 2.7 (used with $k+1, i, p+1$ in place of $n, k, p$ ) insures that $p+1$ divides $l c m(i)\left(\begin{array}{c}k+1 \\ i\end{array}\right)$. Now, for such $i$ 's, the induction hypothesis (on $\left.p\right)$ insures that $l c m(i)$ divides $a_{i}$. Thus, $p+1$ divides $a_{i}\left(\begin{array}{c}k+1 \\ i\end{array}\right)$.

Since $p+1$ divides each one of these three sums, it must divide the last summand $a_{k+1}$.

This finishes the proof of Claim 2 hence of implication $(i) \Rightarrow($ ii $)$ in Theorem 2.5

\subsubsection{Proof of (ii) $\Rightarrow$ (i)}

If $f$ satisfies (ii), then it can be written in the form $f(n)=\sum_{k=0}^{n} b_{k} l c m(k)\left(\begin{array}{l}n \\ k\end{array}\right)$. Consequently,

$$
f(a)-f(b)=\left(\sum_{k=0}^{b} b_{k} l c m(k)\left(\left(\begin{array}{l}
a \\
k
\end{array}\right)-\left(\begin{array}{l}
b \\
k
\end{array}\right)\right)\right)+\sum_{k=b+1}^{a} b_{k} l c m(k)\left(\begin{array}{l}
a \\
k
\end{array}\right)
$$

By Lemma 2.9 $a-b$ divides each term of the first sum.

Consider the terms of the second sum. 
If $a-b \leq b+1$ then $a-b$ divides each term of the second sum since $a-b$ divides $l c m(k)$ for every $k \geq(a-b)$.

If $a-b>b+1$ then, for $b+1 \leq k \leq a$ we have $0 \leq a-k<a-b \leq a$ and Lemma 2.7 (used with $a, k, a-b$ in place of $n, k, p)$ insures that $a-b$ divides $l c m(k)\left(\begin{array}{l}a \\ k\end{array}\right)$. Again, $a-b$ divides each term of the second sum.

\section{Examples of functions having integral differ- ence ratios}

Back to the motivations given in $\$ 1.2$ it may not be obvious to find functions $f$ such that for every finite set $L \subset \mathbb{N}$, the smallest lattice of subsets of $\mathbb{N}$ containing $L$ and closed under $S u c^{-1}$ is also closed under $f^{-1}$. Our characterization by integral difference ratios (Theorem 1.1) gives a first simple class of such functions: polynomial functions. Now, are there non polynomial such functions expressible with usual mathematical functions? It turns out that this is the case and can be proved using the characterization given by Theorem 2.5 for instance, the function such that $f(0)=1, f(x)=\lfloor e x !\rfloor$ for $x \geq 1$ (Theorem 3.1) and variations thereof (e.g. Corollary 3.5).

\subsection{Main examples: around the factorial function}

A simple application of Corollary 2.6 gives functions $\mathbb{N} \rightarrow \mathbb{Z}$ having integral difference ratios with unexpectedly simple analytic expressions up to the ceil and floor functions $\mathbb{R} \rightarrow \mathbb{Z}$.

Theorem 3.1. Let $e$ be the usual Neper constant. For $a \in \mathbb{Z} \backslash\{0,1\}$, the following functions $\mathbb{N} \rightarrow \mathbb{Z}$ have integral difference ratios:

$$
\begin{aligned}
& \phi_{a}^{-}: x \mapsto\left\lfloor e^{1 / a} a^{x} x !\right\rfloor \quad \phi_{a}^{+}: x \mapsto\left\lceil e^{1 / a} a^{x} x !\right\rceil \\
& \phi_{1}^{-}: x \mapsto\left\{\begin{array}{ll}
1 & \text { if } x=0 \\
\lfloor e x !\rfloor & \text { if } x \in \mathbb{N} \backslash\{0\}
\end{array} \quad \phi_{1}^{+}: x \mapsto \begin{cases}2 & \text { if } x=0 \\
\lceil e x !\rceil & \text { if } x \in \mathbb{N} \backslash\{0\}\end{cases} \right.
\end{aligned}
$$

Remark 3.2. Functions $\lfloor e x !\rfloor$ and $\lceil e x !\rceil$ do not have integral difference ratios (cf. Proposition 4.21).

Proof. Recall Taylor-Lagrange formula applied to $t \mapsto e^{t}$ (considered as a map on $\mathbb{R})$ : for all $t \in \mathbb{R}$,

$$
e^{t}=\left(\frac{1}{0 !}+\frac{t}{1 !}+\frac{t^{2}}{2 !}+\cdots+\frac{t^{k-1}}{(k-1) !}+\frac{t^{k}}{k !}\right)+e^{\theta t} \frac{t^{k+1}}{(k+1) !}
$$

for some $0<\theta<1$ depending on $k$ and $t$.

For $a \in \mathbb{Z}$, let $f_{a}: \mathbb{N} \rightarrow \mathbb{Z}$ be the function associated to the Newton series

$$
f_{a}(x)=\sum_{n \in \mathbb{N}} a^{n} n !\left(\begin{array}{l}
x \\
n
\end{array}\right)
$$


Corollary 2.6 insures that $f_{a}$ has integral difference ratios. Moreover,

$$
\begin{aligned}
f_{a}(x)= & \sum_{k \in \mathbb{N}} a^{n} n !\left(\begin{array}{l}
x \\
n
\end{array}\right) \\
= & a^{x} x !\left(\frac{(1 / a)^{x}}{x !}+\frac{(1 / a)^{x-1}}{(x-1) !}+\cdots+\frac{(1 / a)^{1}}{1 !}+\frac{(1 / a)^{0}}{0 !}\right) \\
= & a^{x} x !\left(e^{1 / a}-e^{\theta / a} \frac{(1 / a)^{x+1}}{(x+1) !}\right) \quad \text { for some } 0<\theta<1 \\
& \text { hence } \quad e^{1 / a} a^{x} x !=f_{a}(x)+\frac{e^{\theta / a}}{a(x+1)}
\end{aligned}
$$

Case $a \geq 2$. For $x \in \mathbb{N}$, we have $0<e^{\theta / a} /(a(x+1))<e^{1 / 2} / 2<1$ and, since $f_{a}(x) \in \mathbb{N}$, equation (5) yields $f_{a}(x)=\left\lfloor e^{1 / a} a^{x} x !\right\rfloor$ and $f_{a}(x)+1=\left\lceil e^{1 / a} a^{x} x !\right\rceil$.

Case $a \leq-1$. For $x \in \mathbb{N}$, we have $\left|\frac{e^{\theta / a}}{a(x+1)}\right|=\frac{e^{-\theta /|a|}}{|a|(x+1)} \leq e^{-\theta /|a|}<1$ and $-1<\frac{e^{\theta / a}}{a(x+1)}<0$. Since $f_{a}(x) \in \mathbb{Z}$, equation (5) yields $f_{a}(x)=\left\lceil e^{1 / a} a^{x} x !\right\rceil$ and $f_{a}(x)-1=\left\lfloor e^{1 / a} a^{x} x !\right\rfloor$.

Case $a=1$. For $x \in \mathbb{N}, x \geq 2$, we have $0<e^{\theta} /(x+1)<e / 3<1$ and, again, equation (5) yields $f_{1}(x)=\lfloor e x !\rfloor$ and $f_{1}(x)+1=\lceil e x !\rceil$. Also, $f_{1}(0)=1<2=\lfloor e 0 !\rfloor, f_{1}(1)=2=\lfloor e 1 !\rfloor$ and $f_{1}(0)+1=2<3=\lceil e 0 !\rceil$, $f_{1}(1)+1=3=\lceil e 0 !\rceil$.

Thus, the functions in the statement of the theorem are among the $f_{a}$ 's, $f_{a}+1$ 's and $f_{a}-1$ 's, all of which have rational difference ratios.

\subsection{Algebra of functions having integral difference ratios and applications}

In order to get variations of Theorem 3.1 we state some closure properties of the family of functions with integral difference ratios: sum, product (i.e. they form a subring of functions from $\mathbb{N}$ to $\mathbb{Z}$ ) and composition.

Proposition 3.3. [Subring] If $f, g: \mathbb{N} \rightarrow \mathbb{Z}$ have integral difference ratios then so have their sum and product.

Proof. For product, use equality $f(x) g(x)-f(y) g(y)=f(x)(g(x)-g(y))+$ $g(y)(f(x)-f(y))$.

Corollary 3.4. Every polynomial with coefficients in $\mathbb{Z}$ defines a function $\mathbb{N} \rightarrow$ $\mathbb{Z}$ having integral difference ratios.

Proof. Observe that the identity and constant functions have integral difference ratios and apply Proposition 3.3

Corollary 3.5. Let $s, a \in \mathbb{Z}, a \neq 0$. Let $h_{a, s}$ be any one of the functions $\left\lfloor s e^{1 / a} a^{x} x !\right\rfloor,\left\lceil s e^{1 / a} a^{x} x !\right\rceil$, with $a \in \mathbb{Z} \backslash\{0\}$. There exists a function $g_{a, s}: \mathbb{N} \rightarrow$ $\mathbb{Z}$ having integral difference ratios such that $h_{a, s}(x)=g_{a, s}(x)$ for all $x \geq$ se -1 . 
Proof. Let $f_{a}$ be as in the proof of Theorem 3.1. Proposition 3.3 insures that the function $g_{a, s}(x)=s f_{a}=s \sum_{k \in \mathbb{N}} a^{k} k !\left(\begin{array}{l}x \\ k\end{array}\right)$ has integral difference ratios. Also, Equation (4) above yields

$$
s e^{1 / a} a^{x} x !=s f_{a}(x)+\frac{s e^{\theta / a}}{a(x+1)}
$$

with $0<\theta<1$. If $x \geq s e-1$ then $\left|s e^{\theta / a} / a(x+1) !\right|<1$ and we can argue as in the proof of Theorem 3.1 to finish the proof.

Example 3.6. The bound $s e-1$ (of Corollary 3.5, obtained by majorizing $\theta$ by 1) may not be optimal. For instance, equalities $s f_{1}(x)=s\lfloor$ e $x !\rfloor=\lfloor$ se $x !\rfloor$ may hold for some $x<s e-1$. For instance, $2 e-1=4.436 \ldots, 3 e-1=7.154 \ldots$. but

$f_{2}(x)=2\lfloor$ e $x !\rfloor=\lfloor 2 e x !\rfloor \quad$ for $x \geq 2$
$f_{2}(0)=2<2\lfloor$ e $0 !\rfloor=4<\lfloor 2 e 0 !\rfloor=5$
$f_{2}(1)=4=2\lfloor e 1 !\rfloor<\lfloor 2 e 1 !\rfloor=5$

$f_{2}(x)=2\lfloor e x !\rfloor=\lfloor 2 e x !\rfloor \quad$ for $x \geq 2$ $f_{2}(0)=2<2[e 0 !]=4<\lfloor 2 e 0 !]=5$

Closure under composition gives more variations of our main example (Theorem 3.1).

Proposition 3.7. [Composition] If $f: \mathbb{N} \rightarrow \mathbb{Z}$ and $g: \mathbb{N} \rightarrow \mathbb{N}$ have integral difference ratios then so has $f \circ g$.

Proof. Use transitivity of divisibility: $x-y$ divides $g(x)-g(y)$ which divides $f(g(x))-f(g(y))$.

The following simple result allows to use Proposition 3.7, to extend the scope of Theorem 3.1

Proposition 3.8. If $f: \mathbb{N} \rightarrow \mathbb{Z}$ is not a constant function and has integral difference ratios then, for every $z \in \mathbb{N}$, the set $f^{-1}(z)$ is finite.

Proof. Suppose $f^{-1}(d)$ is infinite for some $d \in \mathbb{Z}$. Consider some $x \in \mathbb{N}$. Then $x-a$ divides $f(x)-d$ for every $a \in f^{-1}(d)$. Thus, $f(x)-d$ has infinitely many divisors hence $f(x)=d$. This shows that $f$ is the constant function with value $d$.

Corollary 3.9. Suppose $g: \mathbb{N} \rightarrow \mathbb{N}$ has integral difference ratios and $a \in \mathbb{Z} \backslash\{0\}$; let $h_{a}: \mathbb{N} \rightarrow \mathbb{Z}$ be such that

$$
\text { if } a \neq 1 \text { then } h_{a}(x)=\left\lfloor e^{1 / a} a^{g(x)} g(x) !\right\rfloor, h_{1}(x)= \begin{cases}1 & \text { if } g(x)=0 \\ \lfloor\text { e } g(x) !\rfloor & \text { if } g(x) \neq 0\end{cases}
$$

1. If $g$ is not constant then $h_{1}$ differs from $\lfloor$ e $g(x) !\rfloor$ on finitely many $x$ 's.

2. For $a \in \mathbb{Z} \backslash\{0\}$ the map $h_{a}$ has integral difference ratios.

Proof. 1. By Proposition $3.8 g^{-1}(0)$ is finite. Hence $h_{1}(x)$ and $\lfloor$ e $g(x) !\rfloor$ differ on finitely many $x$ 's.

2. Use Theorem 3.1 and Proposition 3.7

Example 3.10. The following functions have integral difference ratios:

$$
x \mapsto\left\{\begin{array}{ll}
\left.\left\lfloor e\left(x^{2}-5 x+6\right) !\right)\right\rfloor & \text { if } x \neq 2,3 \\
1 & \text { if } x=2,3
\end{array} \quad, \quad x \mapsto \begin{cases}\lfloor e\lfloor e x !\rfloor !\rfloor & \text { if } x \neq 0 \\
2 & \text { if } x=0\end{cases}\right.
$$




\subsection{Examples with generalized hyperbolic functions}

The proof of Theorem 3.1 can be extended to get more functions having integral difference ratios and which are finite modifications of functions around the factorial functions. Namely, for any given period $k \geq 2$ and any $a \in \mathbb{Z} \backslash\{0\}$, there exist real numbers $\alpha_{0}, \ldots, \alpha_{k-1}$ such that the function $g$ defined by $g(x)=\left\lfloor\alpha_{s} a^{x} x !\right\rfloor$ for $x \in s+k \mathbb{N}$ has integral difference ratios. The main examples (Theorem 3.1) correspond to the (here excluded) degenerate case $k=1$.

First, we need simple results about the generalized hyperbolic functions (a notion which goes back to V. Ricatti, 1754, for instance cf. [24, 13]).

Definition 3.11. Let $\gamma \in \mathbb{R}$. For $k, r \in \mathbb{N}$ such that $k \geq 2$ and $r<k$, the $\gamma$-hyperbolic function $F_{k, r}^{\gamma}: \mathbb{R} \rightarrow \mathbb{R}$ is defined as follows: for $t \in \mathbb{R}$,

$$
F_{k, r}^{\gamma}(t)=\sum_{n \in k \mathbb{N}+r} \gamma^{\lfloor n / k\rfloor} \frac{t^{n}}{n !}=\sum_{n \in \mathbb{N}} \gamma^{n} \frac{t^{k n+r}}{(k n+r) !}
$$

(so that $F_{2,0}^{1}=\cosh , F_{2,1}^{1}=\sinh , F_{2,0}^{-1}=\cos , F_{2,1}^{-1}=\sin$ are the usual hyperbolic and trigonometric functions).

Recall some properties of the 1-hyperbolic functions.

Lemma 3.12. Let $k, r \in \mathbb{N}$ be such that $k \geq 2$ and $r<k$.

1. If $t \neq 0$ and $-1<t<1$ then the sign of $F_{k, r}^{1}(t)$ is that of $t^{r}$ and

$$
0<\frac{F_{k, r}^{1}(t)}{t^{r}} \leq \cosh (|t|)<\cosh (1)=1.543 \ldots
$$

2. Let $\omega=e^{2 i \pi / k}$ be the canonical primitive $k$-th root of unity in the complex plane. For all $t \in \mathbb{R}$,

$F_{k, r}^{1}(t)=\frac{1}{k} \sum_{\ell=0}^{\ell=k-1} \omega^{-\ell r} e^{\omega^{\ell} t}=\frac{1}{k} \sum_{\ell=0}^{\ell=k-1} e^{t \cos \left(\ell r \frac{2 \pi}{k}\right)} \cos \left(-\ell r \frac{2 \pi}{k}+t \sin \left(\ell \frac{2 \pi}{k}\right)\right)$

3. For $q \geq 1$, the $q$-th derivative of $F_{k, r}^{1}$ is $\left(F_{k, r}^{1}\right)^{(q)}=F_{k, s}^{1}$, where $0 \leq s<k$ and $s \equiv r-q(\bmod k)$.

Proof. 1. For $-1<t<1, t \neq 0$, we have

$$
\frac{F_{k, r}^{1}(t)}{t^{r}}=\sum_{n \in \mathbb{N}} \frac{t^{k n}}{(k n+r) !}=\sum_{n \in \mathbb{N}}\left(\frac{|t|^{2 n k}}{(2 n k+r) !}+\varepsilon \frac{|t|^{(2 n+1) k}}{((2 n+1) k+r) !}\right)
$$

where $\varepsilon=1$ if $t>0$ or $k$ is even and $\varepsilon=-1$ if $t<0$ and $k$ is odd.

Since $k \geq 2$ we have $((2 n+1) k+r) !>(2 n k+r)$ ! and since $|t|<1$ and $t \neq 0$ we have $|t|^{2 n k}>|t|^{(2 n+1) k}$. In particular, for both possible values of $\varepsilon$, the last sum in (7) consist of strictly positive terms hence $F_{k, r}^{1}(t) / t^{r}$ is strictly positive. Also, since $k \geq 2$ and $r \geq 0$,

$$
\begin{aligned}
& \frac{F_{k, r}^{1}(t)}{t^{r}} \leq \sum_{n \in \mathbb{N}}\left(\frac{|t|^{2 n k}}{(2 n k+r) !}+\frac{|t|^{(2 n+1) k}}{((2 n+1) k+r) !}\right) \\
\leq & \sum_{n \in \mathbb{N}}\left(\frac{|t|^{4 n}}{(4 n) !}+\frac{|t|^{4 n+2}}{(4 n+2) !}\right)=\sum_{m \in \mathbb{N}} \frac{|t|^{2 m}}{(2 m) !}=\cosh (|t|)<\cosh (1)=1.543 \ldots .
\end{aligned}
$$


2. Arguing in the complex plane, for $\ell=0, \ldots, k-1$ and $t \in \mathbb{R}$ and $r=$ $0, \ldots, k-1$, we have

$$
\begin{gathered}
e^{\omega^{\ell} t}=\sum_{n \in \mathbb{N}} \frac{\omega^{\ell n} t^{n}}{n !}=\sum_{u=0}^{u=k-1} \sum_{m \in \mathbb{N}} \omega^{\ell(k m+u)} \frac{t^{k m+u}}{(k m+u) !}=\sum_{u=0}^{u=k-1} \omega^{\ell u} F_{k, u}^{1}(t) \\
\frac{1}{k} \sum_{\ell=0}^{\ell=k-1} \omega^{-\ell r} e^{\omega^{\ell} t}=\frac{1}{k} \sum_{u=0}^{u=k-1}\left(\sum_{\ell=0}^{\ell=k-1} \omega^{\ell(u-r)}\right) F_{k, u}^{1}(t)=F_{k, r}^{1}(t)
\end{gathered}
$$

since $\sum_{\ell=0}^{\ell=k-1} \omega^{\ell(u-r)}$ is equal to $k$ for $u=r$ and equal to 0 for $u \in\{0, \ldots, k-$ $1\} \backslash\{r\}$. Now, since $t \in \mathbb{R}$ so is $F_{k, r}^{1}(t)$ hence $F_{k, r}^{1}(t)$ is equal to the real part of the above expression in the complex plane. To conclude, observe that

$$
\begin{aligned}
\omega^{-\ell r} e^{\omega^{\ell} t}=e^{i(-\ell r 2 \pi / k)} e^{t \cos (\ell 2 \pi / k)+i t(\sin (\ell 2 \pi / k))} & \\
& =e^{t \cos (\ell 2 \pi / k)} e^{i(-\ell r 2 \pi / k+t \sin (\ell 2 \pi / k))} .
\end{aligned}
$$

3. Using the definition of $F_{k, r}^{1}$ as a series, the derivative of $F_{k, r}^{1}$ is

$$
\left(F_{k, r}^{1}\right)^{\prime}(t)=\sum_{n \in k \mathbb{N}+r, n \geq 1} \frac{t^{n-1}}{(n-1) !}= \begin{cases}F_{k, r-1}^{1}(t) & \text { if } r \geq 1 \\ F_{k, k-1}^{1}(t) & \text { if } r=0\end{cases}
$$

An obvious induction on $q$ concludes the proof.

Theorem 3.13. For any $a \in \mathbb{Z} \backslash\{0\}, k \in \mathbb{N} \backslash\{0,1\}, r \in\{0, \ldots, k-1\}$, let $\mathcal{F}_{a, k, r}$ and $\mathcal{C}_{a, k, r}$ be the following functions $\mathbb{N} \rightarrow \mathbb{Z}$ :

$$
\begin{aligned}
& \mathcal{F}_{a, k, r}(x) \\
& \left\{\begin{array}{ccc}
\left\lfloor F_{k, 0}^{1}(1 / a) a^{x} x !\right\rfloor & \text { if } x \in k \mathbb{N}+r & \left\lceil F_{k, 0}^{1}(1 / a) a^{x} x !\right\rceil \\
\vdots & \vdots & \vdots \\
\left\lfloor F_{k, k-1}^{1}(1 / a) a^{x} x !\right\rfloor & \text { if } x \in k \mathbb{N}+r+k-1 & \left\lceil F_{k, k-1}^{1}(1 / a) a^{x} x !\right\rceil
\end{array}\right\}
\end{aligned}
$$

Let us denote $f \oplus\left\{\left(0, n_{0}\right), \ldots,\left(\ell, n_{\ell}\right)\right\}$ the function $g$ such that $g(x)=f(x)$ if $x>\ell$ and $g(t)=n_{t}$ if $0 \leq t \leq \ell$.

The following functions $\mathbb{N} \rightarrow \mathbb{Z}$ have integral difference ratios:

- Case $|a| \geq 2$ and $r=0 . \mathcal{F}_{a, k, 0}$ and $\mathcal{C}_{a, k, 0}$,

- Case $1 \leq r<k$ and either $a \geq 2$ or $a \leq-2$ and $k-r$ is even. $\mathcal{F}_{a, k, r} \oplus\{(0,0), \ldots(r-1,0)\}$ and $\mathcal{C}_{a, k, r} \oplus\{(0,1), \ldots(r-1,1)\}$,

- Case $1 \leq r<k$ and $a \leq-2$ and $k-r$ is odd. $\mathcal{F}_{a, k, r} \oplus\{(0,-1), \ldots(r-1,-1)\}$ and $\mathcal{C}_{a, k, r} \oplus\{(0,0), \ldots(r-1,0)\}$,

- Case $a=1$ and $r=0 . \mathcal{F}_{1, k, 0} \oplus\{(0,1)\}$ and $\mathcal{C}_{1, k, 0} \oplus\{(0,2)\}$,

- Case $a=1$ and $1 \leq r<k$. $\mathcal{F}_{1, k, r} \oplus\{(0,0) \ldots(r-1,0)\}$ and $\mathcal{C}_{a, k, r} \oplus\{(0,1) \ldots(r-1,1)\}$,

- Case $a=-1$ and $r=0$ and $k$ is even. $\mathcal{F}_{-1, k, 0} \oplus\{(0,0)\}$ and $\mathcal{C}_{-1, k, 0} \oplus\{(0,1)\}$,

- Case $a=-1$ and $r=0$ and $k$ is odd. $\mathcal{F}_{-1, k, 0} \oplus\{(0,1)\}$ and $\mathcal{C}_{-1, k, 0} \oplus\{(0,0)\}$,

- Case $a=-1$ and $1 \leq r<k$ and $k$ is even. $\mathcal{F}_{-1, k, r} \oplus\{0,0) \ldots(r-1,0\}$ and $\mathcal{C}_{a, k, r} \oplus\{(0,1) \ldots(r-1,1)\}$. 
- Case $a=-1$ and $1 \leq r<k$ and $k$ is odd.

$\mathcal{F}_{-1, k, r} \oplus\{(0,-1) \ldots(r-1,-1)\}$ and $\mathcal{C}_{a, k, r} \oplus\{(0,0) \ldots(r-1,0)\}$.

We first give an example, then we will prove the Theorem.

Example 3.14. The functions corresponding to $a=k=2$ are

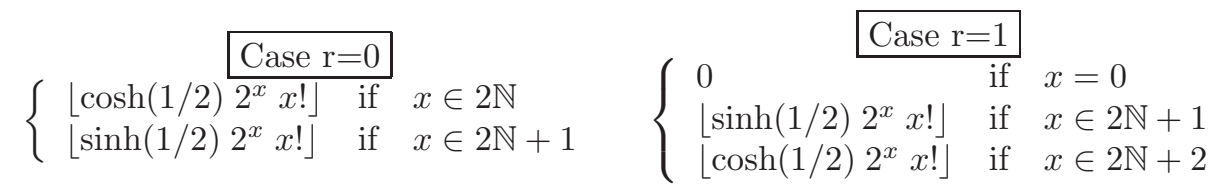

The coefficients $F_{3, r}^{1}(1 / 3)$ occurring in the functions corresponding to $a=k=3$ are given by the following formulas

$$
\begin{aligned}
& F_{3,0}^{1}(t)=(1 / 3)\left(e^{t}+2 e^{-t / 2} \cos (t \sqrt{3} / 2)\right) \\
& F_{3,1}^{1}(t)=(1 / 3)\left(e^{t}-2 e^{-t / 2} \cos (t \sqrt{3} / 2+\pi / 3)\right) \\
& F_{3,2}^{1}(t)=(1 / 3)\left(e^{t}-2 e^{-t / 2} \cos (t \sqrt{3} / 2-\pi / 3)\right)
\end{aligned}
$$

Proof of Theorem 3.13. Since $F_{k, s}^{1}(t)=\sum_{n \in \mathbb{N}} t^{k n+s} /(k n+s)$ ! For $s \in\{0, \ldots, k-$ $1\}$, we have

$$
F_{k, s}^{1}(0)=\sum_{n \in \mathbb{N}} 0^{k n+s} /(k n+s) != \begin{cases}1 & \text { if } s=0 \\ 0 & \text { if } 1 \leq s<k\end{cases}
$$

Since the $q$-th derivative of $F_{k, s}^{1}$ is $F_{k, s^{\prime}}^{1}$ with $0 \leq s^{\prime}<k$ and $s^{\prime} \equiv s-q$ $(\bmod k)($ cf. Lemma 3.12), we have

$$
\left\{\begin{array}{l}
\left(F_{k, s}^{1}\right)^{(q)}(0)=1 \text { if } q \in k \mathbb{N}+s \\
\left(F_{k, s}^{1}\right)^{(q)}(0)=0 \text { otherwise }
\end{array}\right.
$$

and $\left(F_{k, s}^{1}\right)^{(k(u+1)+s)}=F_{k, 0}^{1}$. Thus, the Taylor-Lagrange development at order $k(u+1)+s-1$, of $F_{k, s}^{1}$ at $t$ is, for some $\left.\theta \in\right] 0,1[$,

$$
\begin{aligned}
F_{k, s}^{1}(t) & =\left(\sum_{q=0}^{q=k(u+1)+s-1} \frac{t^{q}}{q !}\left(F_{k, s}^{1}\right)^{(q)}(0)\right)+\frac{t^{k(u+1)+s}}{(k(u+1)+s) !} F_{k, 0}^{1}(\theta t) \\
& =\left(\sum_{m=0}^{m=u} \frac{t^{k m+s}}{(k m+s) !}\right)+\frac{t^{k(u+1)+s}}{(k(u+1)+s) !} F_{k, 0}^{1}(\theta t) .
\end{aligned}
$$

For $k, r \in \mathbb{N}$ such that $k \geq 2$ and $0 \leq r<k$, let $f_{a, k, r}: \mathbb{N} \rightarrow \mathbb{Z}$ be the function associated to the Newton series

$$
f_{a, k, r}(x)=\sum_{n \in k \mathbb{N}+r} a^{n} n !\left(\begin{array}{l}
x \\
n
\end{array}\right) .
$$

By Corollary 2.6, $f_{a, k, r}$ has integral difference ratios. Recall that $\left(\begin{array}{l}x \\ n\end{array}\right)=0$ for $n>x$. Thus,

$$
f_{a, k, r}(x)=0 \quad \text { if } 0 \leq x<r
$$


Also, for $u \in \mathbb{N}, s \in\{0, \ldots, k-1\}$ and $x=u k+r+s$, we have

$$
\begin{aligned}
& f_{a, k, r}(x)=\sum_{p=0}^{p=u} a^{p k+r}(p k+r) !\left(\begin{array}{c}
u k+r+s \\
p k+r
\end{array}\right)=a^{x} x ! \sum_{p=0}^{p=u} \frac{(1 / a)^{x-p k-r}}{(x-p k-r) !} \\
& =a^{x} x ! \sum_{p=0}^{p=u} \frac{(1 / a)^{k(u-p)+s}}{(k(u-p)+s) !} \quad=a^{x} x ! \sum_{m=0}^{m=u} \frac{(1 / a)^{k m+s}}{(k m+s) !}
\end{aligned}
$$

Using equation (8) with $t=1 / a$, we get, for $x=u k+r+s$,

$$
\begin{aligned}
f_{a, k, r}(x) & =a^{x} x ! F_{k, s}^{1}(1 / a)-a^{x} x ! \frac{(1 / a)^{k(u+1)+s}}{(k(u+1)+s) !} F_{k, 0}^{1}(\theta / a) \\
\text { and letting } \Delta & =a^{x} x ! F_{k, s}^{1}(1 / a)-f_{a, k, r}(x) \\
\text { we have } \Delta & =a^{x} x ! \frac{(1 / a)^{k(u+1)+s}}{(k(u+1)+s) !} F_{k, 0}^{1}(\theta / a) \\
& =\frac{F_{k, 0}^{1}(\theta / a)}{a^{k-r} \prod_{j=r+1}^{j=k} k u+s+j}
\end{aligned}
$$

Since $a \in \mathbb{Z} \backslash\{0\}$, we have $0<|\theta / a|<1$. Also, $(\theta / a)^{0}=1$ and point 1 of Lemma 3.12 yields

$$
0<F_{k, 0}^{1}(\theta / a)<\cosh (1)=1.543 \ldots .
$$

Since $x=k u+r+s$, we have $k u+s+j=x+1$ for $j=r+1$. Inequalities (13) and $k-r \geq 1$ insure that

$$
0<|\Delta|<\frac{1.543 \ldots}{|a|^{k-r}(x+1)}<\frac{1.543 \ldots}{|a|(x+1)} .
$$

Equation (12) shows that the sign of $\Delta$ is that of $a^{k-r}$.

Since equation (10) gives $f_{a, k, r}(x)$ for $0 \leq x<r$, it suffices to consider the values $x \geq r$.

Case $a \geq 2$ and Case $a \leq-2$ and $k-r$ even. For every $x \in \mathbb{N}$ we have $0<\Delta<1$. Since $f_{a, k, r}(x) \in \mathbb{Z}$, the definition of $\Delta$ given by (11) yields

$$
\text { for } x \in k \mathbb{N}+r+s\left\{\begin{aligned}
f_{a, k, r}(x) & =\left\lfloor a^{x} x ! F_{k, s}^{1}(1 / a)\right\rfloor \\
f_{a, k, r}(x)+1 & =\left\lceil a^{x} x ! F_{k, s}^{1}(1 / a)\right\rceil
\end{aligned}\right.
$$

Case $a \leq-2$ and $k-r$ odd. For every $x \in \mathbb{N}$ we have $-1<\Delta<0$ hence

$$
\text { for } x \in k \mathbb{N}+r+s\left\{\begin{array}{r}
f_{a, k, r}(x)=\left\lceil a^{x} x ! F_{k, s}^{1}(1 / a) \mid\right. \\
f_{a, k, r}(x)-1=\left\lfloor a^{x} x ! F_{k, s}^{1}(1 / a)\right\rfloor
\end{array}\right.
$$

Case $a=1$ and Case $a=-1$ and $k-r$ even. Then $0<\Delta<1$ for all $x \geq 1$ hence (14) holds with the extra hypothesis $x \geq 1$.

Case $a=-1$ and $k-r$ odd. Then $0<\Delta<1$ for all $x \geq 1$ hence (15) holds with the extra hypothesis $x \geq 1$. 
In both cases $a=1$ and $a=-1$, we also have

$$
f_{-1, k, r}(0)=f_{1, k, r}(0)= \begin{cases}1 & \text { if } r=0 \\ 0 & \text { if } 1 \leq r<k\end{cases}
$$

Thus, the functions mentioned in the theorem are among the functions $f_{a, k, r}$, $f_{a, k, r}-1$ and $f_{a, k, r}+1$, all of which have integral difference ratios.

Open problem. Theorems 3.1 and 3.13, give simple analytic expressions for the functions $\mathbb{N} \rightarrow \mathbb{Z}$ associated to Newton series

$$
\sum_{n \in \mathbb{N}} a^{n} n !\left(\begin{array}{l}
x \\
n
\end{array}\right), \sum_{n \in k \mathbb{N}+r} a^{n} n !\left(\begin{array}{l}
x \\
n
\end{array}\right)
$$

for $a \in \mathbb{Z} \backslash\{0\}$. Theorem 2.5 invites to look at other natural Newton series such as

$$
\sum_{n \in \mathbb{N}} l c m(n)\left(\begin{array}{l}
x \\
n
\end{array}\right), \sum_{n \in k \mathbb{N}+r} l c m(n)\left(\begin{array}{l}
x \\
n
\end{array}\right) .
$$

Is it possible to give analytic expressions to the associated functions?

\subsection{Asymptotic equivalence}

A simple consequence of Theorem 2.5 insures that any function which grows fast enough is asymptotically equivalent to a function having integral difference ratios.

Theorem 3.15. For every function $f: \mathbb{N} \rightarrow \mathbb{Z}$ there exists some function $g: \mathbb{N} \rightarrow \mathbb{Z}$ which has integral difference ratios and such that, for all $x \in \mathbb{N}$,

$$
0 \leq f(x)-g(x) \leq 2^{x} \operatorname{lcm}(x) .
$$

In particular, if there is some $\varepsilon>0$ such that $|f(x)| \geq(2 e+\varepsilon)^{x}$ for all $x$ large enough then $\lim _{x \rightarrow+\infty} \frac{f(x)}{g(x)}=1$, i.e. $f$ and $g$ are asymptotically equivalent.

Proof. Consider the Newton coefficients $\left(a_{k}\right)_{k \in \mathbb{N}}$ of $f$ (cf. Proposition 2.2). Let $a_{k}=l c m(k) q_{k}+b_{k}$ where $0 \leq b_{k}<l c m(k)$ and set $\widetilde{a_{k}}=l c m(k) q_{k}$. Since $l c m(k)$ divides $\widetilde{a_{k}}$ for all $k$ 's, the function $g(x)=\sum_{k \in \mathbb{N}} \widetilde{a_{k}}\left(\begin{array}{l}x \\ k\end{array}\right)$ has integral difference ratios. Also, $0 \leq f(x)-g(x)=\sum_{k \leq x} b_{k}\left(\begin{array}{l}x \\ k\end{array}\right)<\sum_{k \leq x} l c m(k)\left(\begin{array}{l}x \\ k\end{array}\right)<$ $\left(\sum_{k \leq x}\left(\begin{array}{l}x \\ k\end{array}\right)\right) \operatorname{lcm}(x)=2^{x} \operatorname{lcm}(x)$ so that $0 \leq\left|1-\frac{g(x)}{f(x)}\right|<\frac{2^{x} l c m(x)}{|f(x)|}$ which tends to 0 when $x$ tends to $+\infty$ thanks to majoration (3) in Remark 2.4 and the assumption on $f$.

Corollary 3.16. For any real numbers $\alpha, a$ such that $a>0$, the functions $x \mapsto\left\lfloor\alpha a^{x} x !\right\rfloor$ and $x \mapsto\left\lceil\alpha a^{x} x !\right\rceil$ from $\mathbb{N}$ to $\mathbb{Z}$ are asymptotically equivalent to some function $g: \mathbb{N} \rightarrow \mathbb{Z}$ having integral difference ratios.

Proof. Stirling's formula insures that $a^{x} x$ ! satisfies the growth condition of Theorem 3.15.

Remark 3.17. Theorem 3.15 shows that there are functions having integral difference ratios which grow arbitrarily fast. In particular, functions growing much faster than the $a^{x} x$ ! with $a \in \mathbb{Z}$. 


\section{Outside the family of functions with integral difference ratios}

As expected, the examples stated in Theorems 3.1 and 3.13 are kind of exceptions: most functions similar to these examples do not have rational difference ratios. Worse, though they are asymptotically equivalent to functions having rational difference ratios (cf. Theorem 3.15) they can not be uniformly approximated by such functions.

In fact, it turns out that proving non uniform closeness is a very manageable approach to prove failure of the integral difference ratios property.

Using a classical result in the theory of distribution modulo one, we give a general negative result for uniform closeness involving a measure zero set of possible exceptions. Then we look at the problem for some particular classes of functions.

\subsection{Uniform closeness}

Definition 4.1. Two functions $f, g: \mathbb{N} \rightarrow \mathbb{R}$ are uniformly close if $f-g$ is bounded, i.e. there exists $M$ such that $|f(x)-g(x)| \leq M$ for all $x \in \mathbb{N}$.

Some straightforward closure properties will be used together with Propositions 3.3 and 3.7

Proposition 4.2. If $\varphi, \psi: \mathbb{N} \rightarrow \mathbb{Z}$ are uniformly close to $f, g: \mathbb{N} \rightarrow \mathbb{Z}$ then $\varphi+\psi($ resp. $k \varphi)$ is uniformly close to $f+g$ (resp. $k f)$ for all $k \in \mathbb{Z}$.

Proposition 4.3. Let $f, g, \varphi, \psi$ be functions $\mathbb{N} \rightarrow \mathbb{R}$. If $\varphi$ is uniformly close to $f$, and $\psi$ differs from $g$ on finitely many points, then $\varphi \circ \psi$ is uniformly close to $f \circ g$.

Proof. Suppose $|\varphi(t)-f(t)| \leq M$ for all $t \in \mathbb{N}$ and $a_{1}, \ldots, a_{p}$ are the points on which $\psi$ differ from $g$. Let $L=\max \left(M, \max _{i=1, \ldots, p}\left|\varphi\left(\psi\left(a_{i}\right)\right)-f\left(g\left(a_{i}\right)\right)\right|\right)$. Then $\left|\varphi\left(\psi\left(a_{i}\right)\right)-f\left(g\left(a_{i}\right)\right)\right| \leq L$ and, for $x \neq a_{1}, \ldots, a_{p},|\varphi(\psi(x))-f(g(x))|=$ $|\varphi(g(x))-f(g(x))| \leq L$.

\subsection{A general negative result for uniform closeness to func- tions having integral difference ratios}

Recall the following classical notion.

Definition 4.4. For $A \in \mathbb{N} \backslash\{0\}$ and $t \in \mathbb{R}$, the $A$-fractional part of $t$ is $\{t\}_{A}=t-A\lfloor t / A\rfloor$, i.e. $\{k A+u\}_{A}=u$ for any $k \in \mathbb{Z}$ and $u \in[0, A[$. The 1-fractional part $\{t\}_{1}$ is simply denoted by $\{t\}$.

Before entering the wanted negative result, we first observe the following fact.

Lemma 4.5. Suppose $\varphi: \mathbb{N} \rightarrow \mathbb{R}$ is uniformly close to some function $f: \mathbb{N} \rightarrow \mathbb{Z}$ such that $n$ divides $f(n)-f(0)$ for all $n \geq 1$. Then, for all $A \in \mathbb{N}$ big enough, the sequence of $A$-fractional parts $\left(\{\varphi(n A)\}_{A}\right)_{n \in \mathbb{N}}$ is not dense in $[0, A]$. 
Proof. Let $g(n)=f(n)-f(0)$. Then $\varphi$ is also uniformly close to $g$ and $n$ divides $g(n)$ for all $n$. Let $M>0$ be such that $|\varphi(n)-g(n)| \leq M$ for all $n \in \mathbb{N}$. Consider any $A \in \mathbb{N}$ such that $A>2 M$. Then $\varphi(n A) \in[g(n A)-M, g(n A)+M]$. Since $n A$ (hence $A$ ) divides $g(n A)$, we have $\{g(n A)+u\}_{A}=u$ for $u \in[0, A[$ and $\{g(n A)-v\}_{A}=\left\{(g(n A)-A+(A-v)\}_{A}=A-v\right.$ for $\left.-v \in\right]-A, 0[$. In particular,

$$
\begin{aligned}
\left\{\{y\}_{A} \mid y \in[g(n A), g(n A)+M]\right. & =[0, M] \\
\left\{\{y\}_{A} \mid y \in[g(n A)-M, g(n A)[\}\right. & =[A-M, A[
\end{aligned}
$$

so that $\{\varphi(n A)\}_{A} \in[0, M] \cup[A-M, A[$ for all $n \in \mathbb{N}$. Since $A>2 M$ we have $M<A-M$ hence the non empty open subinterval $] M, A-M[$ of $[0, A[$ contains no point of the sequence $\left(\{\varphi(n A)\}_{A}\right)_{n \in \mathbb{N}}$.

We shall use a result from the theory of uniform distribution modulo one.

Definition 4.6. A sequence $\left(t_{n}\right)_{n \in \mathbb{N}}$ is uniformly distributed modulo one if, for all $0 \leq a<b \leq 1$, the proportion of $i$ 's in $\{0, \ldots, n-1\}$ such that the 1 -fractional part $\left\{t_{i}\right\}$ is in $\left[a, b\left[\right.\right.$ tends to $b-a$ when $n$ tends to $+\infty$, i.e. $\lim _{n \rightarrow+\infty} \frac{1}{n} \operatorname{card}\{i \in$ $\{0, \ldots, n-1\} \mid\left\{t_{i}\right\} \in[a, b[\}=b-a$.

Theorem 4.7 (Koksma, 1935, cf. Corollary 4.3 in 11]). Let $\left(\lambda_{n}\right)_{n \in \mathbb{N}}$ be a sequence of reals such that $\inf \left\{\left|\lambda_{m}-\lambda_{n}\right| \mid m \neq n\right\}>0$. Then, for almost all real numbers $\alpha$, the sequence $\left(\alpha \lambda_{n}\right)_{n \in \mathbb{N}}$ is uniformly distributed modulo one.

Remark 4.8. The "almost everywhere" restriction cannot be removed in Theorem 4.7. It is known (cf. Example 4.2 in 11]) that if $t$ is a Pisot-Vijayaraghavan number (in particular, if $t$ is a rational number or $t$ is the golden number $(1+\sqrt{5}) / 2)$ then the sequence $\left(t^{n}\right)_{n \in \mathbb{N}}$ has no limit point except possibly 0 or 1 hence is not uniformly distributed modulo one.

We now come to the wanted general negative result.

Theorem 4.9 (Almost everywhere negative result). Let $\left(\lambda_{n}\right)_{n \in \mathbb{N}}$ be a sequence of real numbers satisfying the condition $\inf \left\{\left|\lambda_{m}-\lambda_{n}\right| \mid m \neq n\right\}>0$. Then, for almost all real numbers $\alpha$, the function $n \mapsto \alpha \lambda_{n}$ is not uniformly close to any function having integral difference ratios.

Proof. The assumed hypothesis on the $\lambda_{n}$ 's, insures that we can apply Koksma's theorem for each sequence $\left(\lambda_{n A} / A\right)_{n \in \mathbb{N}}$ with $A \in \mathbb{N} \backslash\{0\}$. Since sets of measure zero are closed under countable union, Koksma's theorem insures that there exists a set $X \subseteq \mathbb{R}$ such that $\mathbb{R} \backslash X$ has measure zero and, for all $\alpha \in X$, all the sequences $\left(\alpha \lambda_{n A} / A\right)_{n \in \mathbb{N}}$, with $A \in \mathbb{N} \backslash\{0\}$, are uniformly distributed modulo one hence are dense in $[0,1[$. Applying the homothety $t \mapsto A t$, we see that, for all $\alpha \in X$,

All the sequences $\left(\alpha \lambda_{n A}\right)_{n \in \mathbb{N}}$, with $A \in \mathbb{N} \backslash\{0\}$, are dense in $[0, A[$.

By way of contradiction, suppose that, for some $x \in X$, the function $n \mapsto$ $\alpha \lambda_{n}$ is uniformly close to some function $f: \mathbb{N} \rightarrow \mathbb{N}$ having integral difference ratios. Observe that $n$ divides $f(n)-f(0)$ for all $n \in \mathbb{N}$, so that we can apply Lemma 4.5 for $A \in \mathbb{N}$ large enough, the sequence $\left(\alpha \lambda_{n A}\right)_{n \in \mathbb{N}}$ is not dense in $[0, A[$. This contradicts property (16). 
Theorem 4.9 shows that the constant $e^{1 / a}$ has a crucial role in Theorem 3.1

Corollary 4.10. 1. Let $f: \mathbb{N} \rightarrow \mathbb{R}$ be a function such that $\inf _{k \leq m<n} \mid f(m)-$ $f(n) \mid>0$ for some $k$. For almost every $\alpha \in \mathbb{R}$, the real-valued function $n \mapsto$ $\alpha f(n)$ is not uniformly close to a function having integral difference ratios.

2. Let $a \in \mathbb{R} \backslash\{0\}$. For almost every $\alpha \in \mathbb{R}$, the $\mathbb{Z}$-valued functions $n \mapsto$

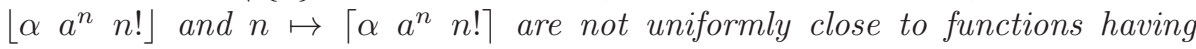
integral difference ratios.

Proof. 1. First modify $f$ on $\{0, \ldots, k\}$ to obtain $f^{\prime}$ such that $\inf _{m<n} \mid f^{\prime}(m)-$ $f^{\prime}(n) \mid>0$. Apply then Theorem 4.9 with $\lambda_{n}=f^{\prime}(n)$, thus $\alpha f^{\prime}$ is not uniformly close to any function having integral difference ratios. The same holds for $\alpha f$ which is equal to $\alpha f^{\prime}$ except on a finite number of points (uniform closeness is not modified by finitely many changes).

2. Applying (1) with $f: n \mapsto a^{n} n$ ! we see that $\alpha f$ is not uniformly close to any $\mathbb{N} \rightarrow \mathbb{Z}$ function having integral difference ratios; the same holds for the $\mathbb{N} \rightarrow \mathbb{Z}$ functions $\left\lfloor\alpha a^{n} n !\right\rfloor$ and $\left\lceil\alpha a^{n} n !\right\rceil$ which are uniformly close to $\alpha f$.

Remark 4.11. 1. Note the difference with Corollary 3.16.

2. In Theorem 3.1, the parameter $a$ is taken in $\mathbb{Z}$ so that the Newton series $\sum_{n \in \mathbb{N}} a^{n} n !\left(\begin{array}{l}x \\ n\end{array}\right)$ takes its values in $\mathbb{Z}$. In the above corollary, we can take $a$ in $\mathbb{R}$.

Example 4.12. Let $P$ be a non constant polynomial with real coefficients; for almost every $\alpha \in \mathbb{R}$, the function $\alpha P$ is not uniformly close to any function having integral difference ratios.

The analog result for the particular constants $F_{k, 0}^{1}(1 / a), \ldots, F_{k, k-1}^{1}(1 / a)$ in Theorem 3.13 requires an easy extension of Theorem 4.9

Theorem 4.13. Let $k, r, s \in \mathbb{N}$ be such that $k \geq 1$ and $0 \leq r<k$. Let $\left(\lambda_{n}\right)_{n \in k \mathbb{N}+r+s}$ be a sequence of real numbers satisfying the condition $\inf \left\{\mid \lambda_{m}-\right.$ $\left.\lambda_{n}|| m, n \in k \mathbb{N}+r+s, m \neq n\right\}>0$. Then, for almost all $\alpha \in \mathbb{R}$, no function $f: \mathbb{N} \rightarrow \mathbb{R}$ such that $f(k x+r+s)=\alpha \lambda_{x}$ for all $x \in \mathbb{N}$ can be uniformly close to some function having integral difference ratios.

Proof. Arguing as in the proof of Theorem 4.9, first, we see that, for all $\alpha \in X$,

All the sequences $\left(\alpha \lambda_{n k A+r+s}\right)_{n \in \mathbb{N}}$, with $A \in \mathbb{N} \backslash\{0\}$, are dense in $[0, A[$

and then we conclude using Lemma 4.5.

Corollary 4.14. Let $a \in \mathbb{R} \backslash\{0\}$ and $k, s \in \mathbb{N}$ be such that $k \geq 2$. For almost all $\alpha \in \mathbb{R}$, every function $f: \mathbb{N} \rightarrow \mathbb{Z}$ such that $f(x)=\left\lfloor\alpha a^{x} x !\right\rfloor$ for all $x \in k \mathbb{N}+s$ has non integral difference ratios. Idem with $\lceil\ldots\rceil$ in place of $\lfloor\ldots\rfloor$.

\subsection{Non integral polynomial functions}

Apart the obvious fact (cf. Corollary 3.4) that polynomials with coefficients in $\mathbb{Z}$ have integral difference ratios, there are only negative results for polynomials with real coefficients. In particular, the positive result with $\left\lfloor e^{1 / a} a^{x} x !\right\rfloor$ (cf. Theorem 3.1) has no analog with polynomials. Theorem 4.15 completes Corollary 3.4. Example 4.12 and Corollary 4.10. 
Theorem 4.15. Let $P(x)=\sum_{i=0}^{i=k} \alpha_{i} x^{i}$, be a polynomial with real coefficients and consider it as a function $\mathbb{N} \rightarrow \mathbb{R}$. The following conditions are equivalent.

(i) The coefficients $\alpha_{i}, i=1, \ldots, k$ of $P$ are in $\mathbb{Z}$.

(ii) $P$ maps $\mathbb{N}$ into $\mathbb{Z}$ and has integral difference ratios.

(iii) $P: \mathbb{N} \rightarrow \mathbb{R}$ is uniformly close to some function $\mathbb{N} \rightarrow \mathbb{Z}$ having integral difference ratios.

Proof. $(i) \Rightarrow(i i)$ is Corollary 3.4 and $(i i) \Rightarrow($ iii $)$ is trivial. We prove $(i i i) \Rightarrow(i)$. Suppose condition (iii) is true; let $N_{a, b}, K, \theta_{x}$ be defined as in points (a) and (b) below.

(a) $\varphi: \mathbb{N} \rightarrow \mathbb{Z}$ has integral difference ratios. For $a, b \in \mathbb{N}, a \neq b$, let $N_{a, b} \in \mathbb{Z}$ be such that $\varphi(a)-\varphi(b)=N_{a, b}(a-b)$,

(b) $P$ is uniformly close to $\varphi$ and $K, \theta_{x}$ are such that, for all $x \in \mathbb{N}, \mid P(x)-$ $\varphi(x) \mid \leq K$, i.e. $P(x)=\varphi(x)+\theta_{x} K$ for some $\theta_{x} \in[-1,1]$.

By induction on the degree of $P$ we prove that all coefficients of $P$, except may be $\alpha_{0}$ are in $\mathbb{Z}$.

Basis: if $P=\alpha_{1} x+\alpha_{0}$ has degree one then $\alpha_{1} \in \mathbb{Z}$. By point (a), $\varphi(x)-$ $\varphi(0)=N_{x} x, N_{x} \in \mathbb{N}$. By point (b), $P(x)-P(0)=\varphi(x)-\varphi(0)+\left(\theta_{x}-\theta_{0}\right) K$, $\theta_{x}, \theta_{0} \in[-1,1]$. Let $\alpha_{1}=\left\lfloor\alpha_{1}\right\rfloor+\theta, \theta \in[-0,1[$. Thus for all $x, P(x)-P(0)=$ $\left\lfloor\alpha_{1}\right\rfloor x+\theta x=N_{x} x+\left(\theta_{x}-\theta_{0}\right) K$, hence $x\left(\left\lfloor\alpha_{1}\right\rfloor-N_{x}+\theta\right)=\left(\theta_{x}-\theta_{0}\right) K$. Assume by contradiction $\alpha_{1} \notin \mathbb{Z}$ and $\theta \neq 0$, and let $x>\frac{3 K}{\min (\theta, 1-\theta)}$ : noting that $\left\lfloor\alpha_{1}\right\rfloor-N_{x} \in \mathbb{Z}$, we have $x\left(\left\lfloor\alpha_{1}\right\rfloor-N_{x}+\theta\right)>3 K$, contradicting $\left(\theta_{x}-\theta_{0}\right) K<2 K$. This show that, if $P$ has degree one, then $\alpha_{1} \in \mathbb{Z}$.

Induction: it suffices to prove that $\alpha_{k}$, the leading coefficient of $P$, is in $\mathbb{Z}$. Then, an induction on the degree of $P$ concludes the proof: if $\alpha_{k} \in \mathbb{Z}$ then $P(x)-\alpha_{k} x^{k}$ also satisfies condition (iii) (by Proposition 4.2) and has degree $k-1$.

An easy way to single out $\alpha_{k}$ is to consider the $k$-th derivative of $P$ which is $k ! \alpha_{k}$. Since we are in a discrete context with functions defined on $\mathbb{N}$ and not on $\mathbb{R}$, we turn to finite differences.

For $1 \leq n \leq k$, define a polynomial $P^{(n)}$ by the following induction:

$$
\begin{aligned}
P^{(1)}(x) & =\frac{P(2 x)-P(x)}{x}=\sum_{i=1}^{i=k} \alpha_{i}\left(2^{i}-1\right) x^{i-1} \\
\text { if } 1<n \leq k \quad P^{(n)}(x) & =\frac{P^{(n-1)}(2 x)-P^{(n-1)}(x)}{x} \\
& =\sum_{i=n}^{i=k} \alpha_{i}\left(\prod_{j=i-n+1}^{j=i}\left(2^{j}-1\right)\right) x^{i-n}
\end{aligned}
$$

Claim 1. For every $n \in\{1, \ldots, k\}$ and $a \in \mathbb{N} \backslash\{0\}$,

$$
P^{(n)}(a)=\frac{M_{a}^{(n)}}{2^{s(n)} a^{n-1}}+\frac{\xi_{a}^{(n)}}{2^{t(n)} a^{n}} \quad \text { with } M_{a}^{(n)} \in \mathbb{Z} \text { and }\left|\xi_{a}^{(n)}\right| \leq K_{n} .
$$

where, for $n \geq 1, s(n)=\frac{(n-1)(n-2)}{2}, t(n)=\frac{(n-1) n}{2}=s(n)+n-1$ and $K_{n}=K \prod_{i=1}^{i=n}\left(2^{i-1}+1\right)$. 
Proof. We argue by induction on $n$. Case $n=1$ is as follows:

$$
\begin{array}{rlrl}
P^{(1)}(a) & =\frac{\varphi(2 a)-\varphi(a)+\left(\theta_{2 a}-\theta_{a}\right) K}{a} & & \text { (cf. point (b) above) } \\
& =N_{2 a, a}+\frac{\left(\theta_{2 a}-\theta_{a}\right) K}{a} & & \text { (cf. point (a) above) } \\
& =M_{a}^{(1)}+\frac{\xi_{a}^{(1)}}{a} & \text { where } M_{a}^{(1)} \in \mathbb{Z} \text { and }\left|\xi_{a}^{(1)}\right| \leq K_{1}=2 K
\end{array}
$$

Induction step $2 \leq n \leq k$.

$$
\begin{aligned}
P^{(n)}(a) & =\frac{P^{(n-1)}(2 a)-P^{(n-1)}(a)}{a} \\
& =\frac{1}{a}\left(\frac{M_{2 a}^{(n-1)}}{2^{s(n-1)}(2 a)^{n-2}}-\frac{M_{a}^{(n-1)}}{2^{s(n-1)} a^{n-2}}+\frac{\xi_{2 a}^{(n-1)}}{2^{t(n-1)}(2 a)^{n-1}}-\frac{\xi_{a}^{(n-1)}}{2^{t(n-1)} a^{n-1}}\right) \\
& =\frac{M_{2 a}^{(n-1)}-2^{n-2} M_{a}^{(n-1)}}{2^{s(n-1)+n-2} a^{n-1}}+\frac{\xi_{2 a}^{(n-1)}-2^{n-1} \xi_{a}^{(n-1)}}{2^{t(n-1)+n-1} a^{n}} \\
& =\frac{M_{a}^{(n)}}{2^{s(n)} a^{n-1}}+\frac{\xi_{a}^{(n)}}{2^{t(n)} a^{n}}
\end{aligned}
$$

with $M_{a}^{(n)} \in \mathbb{Z}$ and $\left|\xi_{a}^{(n)}\right| \leq K_{n}=\left(2^{n-1}+1\right) K_{n-1}$.

Claim 2. There exist integers $L, T \geq 2$ such that, for every $a \in \mathbb{N}, a \geq 1$,

$$
\alpha_{k}=\left\lfloor\alpha_{k}\right\rfloor+\frac{N_{a}}{(L a)^{k-1}}+\frac{\eta_{a}}{(L a)^{k}}
$$

for some $N_{a} \in\{0,1, \ldots, L a-1\}$ and some real $\eta_{a} \in[-T, T]$.

Proof. Since $P^{(k)}(x)$ is the constant polynomial $\alpha_{k} \ell$ where $\ell=\prod_{j=1}^{j=k}\left(2^{j}-1\right)$, Claim 1 yields, writing the representation in base $L a$ of $\alpha_{k}-\left\lfloor\alpha_{k}\right\rfloor$ for $L=$ $\ell 2^{s(k)+k-1}$,

$$
\alpha_{k}=\frac{M_{a}^{(k)}}{\ell 2^{s(k)} a^{k-1}}+\frac{\xi_{a}^{(k)}}{\ell 2^{s(k)+k-1} a^{k}}=\left\lfloor\alpha_{k}\right\rfloor+\frac{N_{a}}{(L a)^{k-1}}+\frac{\eta_{a}}{(L a)^{k}}
$$

where $T=L^{k-1} K_{k}, \eta_{a}=L^{k-1} \xi_{a}^{(k)}, N_{a}=L^{k-2} 2^{(k-1)^{2}} M_{a}^{(k)}$. Clearly, $\left|\eta_{a}\right| \leq$ $T$.

Claim 3. Suppose $k \geq 2$. For every $b \in \mathbb{N}, b>\frac{T}{L}$, the real $\alpha_{k}$ is in

$$
\frac{\mathbb{Z}}{(L b)^{\mathbb{N}}}=\left\{\frac{p}{(L b)^{n}} \mid p \in \mathbb{Z}, n \in \mathbb{N}\right\} .
$$

Proof. For any $s, i \in \mathbb{N}$ such that $s \geq 2$ and $i \geq 1$, let $d(s, i) \in\{0,1, \ldots, s-1\}$ be the $i$-th digit of the representation in base $s$ of the real $\alpha_{k}-\left\lfloor\alpha_{k}\right\rfloor$. In case this real is in $\mathbb{Z} / s^{\mathbb{N}}$, choose the representation which ends by an infinite tail of 0's. 
Observe that the digit $d\left(s^{p}, k\right)$ (which is in $\left\{0, \ldots, s^{p}-1\right\}$ ) is represented in base $s$ by the length $p$ sequence of digits $d(s, i)$ (lying in $\{0, \ldots, s-1\}$ ) for $p(k-1)<i \leq p k$.

Claim 2 insures that if $a \in \mathbb{N}$ satisfies $L a>T$ then, in base $L a$, the $k$-th digit $d(L a, k)$ of $\alpha_{k}-\left\lfloor\alpha_{k}\right\rfloor$ is either in $\{0, \ldots, T\}$ (case $\eta_{a} \in[0, T]$ ) or is in $\{L a-T, \ldots, L a-1\}$ (case $\eta_{a} \in[-T, 0[)$.

Let $a=L^{p-1} b^{p}$ where $p \geq 2$ and $L b>T$. Then $L a=(L b)^{p}$ and the digit $d(L a, k)$ which lies in $\{0, \ldots, T\} \cup\{L a-T, \ldots, L a-1\}$ is represented in base $L b$ by one of the two length $p$ sequences

$00 \ldots 0 \lambda \quad($ with $\lambda \in\{0, \ldots, T\}) \quad, \quad \delta \delta \ldots \delta \mu \quad($ with $\mu \in\{L a-T, \ldots, L a-1\})$

In particular, going from base $L a=(L b)^{p}$ to base $L b$, the $p-1$ digits $d(L b, i)$, for $p(k-1)<i<p k$, are all 0 or are all $\delta$. Observe that, for $p>k+1$ we have $(p+1)(k-1)+1<p k-1$ hence the two sets $\{p(k-1)+1, \ldots, p k-1\}$ and $\{(p+1)(k-1)+1, \ldots,(p+1) k-1\}$ have non empty intersection. As a consequence, the base $L b$ digits $d(L b, i), i>(k+1)(k-1)+1=k^{2}$, are all 0 or are all $\delta$. Thus, the real $\alpha_{k}-\left\lfloor\alpha_{k}\right\rfloor$ is in $\frac{\mathbb{Z}}{(L b)^{\mathbb{N}}}$. And so is the real $\alpha_{k}$.

Claim 4. If $k \geq 2$ then the real $\alpha_{k}$ is in $\mathbb{Z}$.

Proof. Choose prime integers $b, c$ such that $b>c>\max (L, T / L)$. Claim 3 insures that $\alpha_{k}$ is in both sets $\frac{\mathbb{Z}}{(L b)^{\mathbb{N}}}$ and $\frac{\mathbb{Z}}{(L c)^{\mathbb{N}}}$. Now, if $p, q \in \mathbb{Z}$ and $m, n \in \mathbb{N}$, equality $\frac{p}{(L b)^{m}}=\frac{q}{(L c)^{n}}$ with $p, q \in \mathbb{Z}$ and $m, n \in \mathbb{N}$, means $p L^{n} c^{n}=q L^{m} b^{m}$. Since $b, c$ are distinct prime larger than $L$, we see that $c^{n}$ divides $q$ and $b^{m}$ divides $p$. In particular, $\frac{p}{(L b)^{m}} \in \mathbb{Z}$. Thus, the intersection of $\frac{\mathbb{Z}}{(L b)^{\mathbb{N}}}$ and $\frac{\mathbb{Z}}{(L c)^{\mathbb{N}}}$ is $\mathbb{Z}$.

This Claim finishes the proof of the theorem.

\subsection{Functions around the exponential functions}

We first apply the general negative result Theorem 4.9.

Theorem 4.16. Let $\beta$ be a real number such that $\beta>1$. For almost all real numbers $\alpha$, the function $\mathbb{N} \rightarrow \mathbb{R}$ such that $n \mapsto \alpha \beta^{n}$ is not uniformly close to any function $\mathbb{N} \rightarrow \mathbb{Z}$ having integral difference ratios.

Proof. Apply Theorem 4.9 with $\lambda_{n}=\beta^{n}$.

For $\beta \in \mathbb{N}$, the above result holds for all $\alpha \neq 0$ rather than almost all $\alpha$.

Theorem 4.17. Let $\alpha$ be a non zero real number and $k \in \mathbb{N} \backslash\{0,1\}$. The function $\mathbb{N} \rightarrow \mathbb{R}$ such that $n \mapsto \alpha k^{n}$ is not uniformly close to any function $\mathbb{N} \rightarrow \mathbb{Z}$ having integral difference ratios.

Assuming $f: \mathbb{N} \rightarrow \mathbb{N}$ is uniformly close to $\alpha k^{x}$, to show that $f$ does not have integral difference ratios, we apply the integral difference ratios assumption to suitably chosen ordered pairs $\langle a, b\rangle$. 
We first consider the case $\alpha \in \mathbb{N} \backslash\{0\}$ and show that, letting $\delta_{x}=f(x)-m k^{x}$, the sequence of deviations $\left(\delta_{x}\right)_{x \in \mathbb{N}}$ is periodic (Lemma 4.18) and this yields a contradiction. For $\alpha \in \mathbb{R} \backslash\{0\}$, using another family of ordered pairs $\langle a, b\rangle$, we then prove that the base $k$ expansion of $\alpha$ is periodic (Lemma 4.19). The proof of Theorem 4.17 is then be easily concluded.

Lemma 4.18. If $k \in \mathbb{N} \backslash\{0,1\}$ and $f: \mathbb{N} \rightarrow \mathbb{N}$ is uniformly close to the function $x \mapsto m k^{x}$, with $m \in \mathbb{N}$, then $f$ does not have integral difference ratios.

Proof. Let us write $f(x)=m k^{x}+\delta_{x}$ with $\delta_{x} \in \mathbb{N}$ such that $\delta_{x}<M$. Let $\mu \in \mathbb{N}$ be such that $M<k^{\mu-1}-1$, hence

$$
\forall x \in \mathbb{N} \quad \delta_{x}=\left|f(x)-m k^{x}\right|<k^{\mu-1}-1
$$

Let us apply the integral difference ratios assumption with $a-b=N\left(k^{N}-1\right)$ for some $N \geq \mu+2$. Then $N\left(k^{N}-1\right)$ divides $f(a)-f(b)$, and in particular,

$$
f(a)-f(b) \equiv 0 \quad\left(\bmod \left(k^{N}-1\right)\right)
$$

Now, $c-1$ divides $c^{d}-1$ for all $c, d \geq 1$. Letting $c=k^{N}$ and $d=k^{N}-1$, we see that

$$
k^{a-b}-1=\left(k^{N}\right)^{k^{N}-1}-1 \equiv 0\left(\bmod \left(k^{N}-1\right)\right) .
$$

Since $f(a)-f(b)=m k^{a}-m k^{b}+\left(\delta_{a}-\delta_{b}\right)=m k^{b}\left(k^{a-b}-1\right)+\left(\delta_{a}-\delta_{b}\right)$, equations (18) and (19) yield

$$
\begin{aligned}
\delta_{a}-\delta_{b} & \equiv 0 \quad\left(\bmod \left(k^{N}-1\right)\right) \\
\text { hence } \quad \delta_{a}-\delta_{b} & =0
\end{aligned}
$$

The sequence $\left(\delta_{x}\right)_{x \in \mathbb{N}}$ is thus periodic, with period $\left(k^{N}-1\right)$. Let $a$ be a multiple of $m k\left(k^{N}-1\right)$. Since $k \geq 2$, inequality (17) yields $\left|\delta_{a}-\delta_{0}\right|<k^{\mu}$. Now, by (20), $\delta_{a}-\delta_{0}$ is divisible by $k^{N}-1>k^{\mu}$. Thus, $\delta_{a}-\delta_{0}=0$, so that $f(a)-f(0)=m\left(k^{a}-1\right)$. This contradicts the integral difference ratios property because $m k$ divides $a$ but does not divide $m\left(k^{a}-1\right)$.

Lemma 4.19. If If $k \in \mathbb{N} \backslash\{0,1\}$ and $f: \mathbb{N} \rightarrow \mathbb{N}$ has integral difference ratios and is uniformly close to the function $x \mapsto \alpha k^{x}$, with $\alpha \in \mathbb{R}$, then $\alpha$ is rational.

Proof. Let $M$ be such that $\left|f(x)-\alpha k^{x}\right|<M$ for all $x \in \mathbb{N}$. For some $\mu \in \mathbb{N}$ we have $M<k^{\mu}$. Let $\ell=k^{\mu+2}$ and $g(x)=f((\mu+2) x)$. Then $g$ also has integral difference ratios and is uniformly close to the function $x \mapsto \alpha \ell^{x}$ and $\left|g(x)-\alpha \ell^{x}\right|<k^{2}=\ell / k^{2} \leq \ell / 4$.

Thus, with no loss of generality, we can reduce to the case $M=k / 4$ with

$$
f(x)=\left\lfloor\alpha k^{x}\right\rfloor+\delta_{x} \quad \text { where } \delta_{x} \in \mathbb{N} \text { satisfies }\left|\delta_{x}\right|<k / 2 \text {. }
$$

We use the base $k$ expansion of integers and reals. In case $\alpha$ is of the form $n / k^{p}$, with $p \in \mathbb{N}$, we systematically consider its infinite base $k$ expansion which ends with a tail of 0 's and not a tail of $(k-1)$ 's. The (finite) base $k$ expansions of the integers $\left\lfloor\alpha k^{b}\right\rfloor$ and $\left\lfloor\alpha k^{a}\right\rfloor$ are related to the (infinite) base $k$ expansion of 
the real $\alpha$. If $w$ is a (finite or infinite) word on the alphabet $\{0,1, \cdots, k-1,$.$\} ,$ we denote by $\bar{w}$ the integer or real having $w$ as base $k$ expansion. Then

$$
\begin{aligned}
\alpha & =\overline{t_{0} t_{1} \ldots t_{p} \cdot t_{p+1} t_{p+2} \cdots} & \\
\left\lfloor\alpha k^{c}\right\rfloor & =\overline{t_{0} t_{1} \ldots t_{p+c}} & \text { (with our convention on tails) } \\
& \equiv t_{p+c} \quad(\bmod k) & \\
\left\lfloor\alpha k^{a}\right\rfloor-\left\lfloor\alpha k^{b}\right\rfloor+\left(\delta_{a}-\delta_{b}\right) & \equiv t_{p+a}-t_{p+b}+\left(\delta_{a}-\delta_{b}\right) & (\bmod k)
\end{aligned}
$$

where the digits $t_{i}$ 's, $i \in \mathbb{N}$, are in $\{0,1, \ldots, k-1\}$ and $\left|\delta_{a}-\delta_{b}\right|<k$.

Let $b \in \mathbb{N}$ and $a=b+k$. The integral difference ratios assumption insures that

$$
\begin{aligned}
& f(a)-f(b) \equiv 0 \quad(\bmod k) \\
& \text { but } \quad f(a)-f(b)=\left\lfloor\alpha k^{a}\right\rfloor-\left\lfloor\alpha k^{b}\right\rfloor+\left(\delta_{a}-\delta_{b}\right) \\
& \text { hence } t_{p+a}-t_{p+b}+\left(\delta_{a}-\delta_{b}\right) \equiv 0 \quad(\bmod k)
\end{aligned}
$$

Since $\left|t_{p+a}-t_{p+b}+\left(\delta_{a}-\delta_{b}\right)\right| \leq 2 k-2$, we see that $t_{p+a}-t_{p+b}+\left(\delta_{a}-\delta_{b}\right) \in$ $\{-k, 0, k\}$. Recalling that $b$ is arbitrary and $a=b+k$, this means that

$$
\forall n \geq p \quad \overline{t_{n} t_{n+1}}-\overline{t_{n+k} t_{n+1+k}} \in\{-k, 0, k\}
$$

To conclude, we argue by cases.

Case $t_{n}=t_{n+k}$ for some $n \geq p$. Then (24) insures that $\overline{t_{n} t_{n+1}}-\overline{t_{n+k} t_{n+1+k}}=0$ hence $t_{n+1}=t_{n+1+k}$ and, via an obvious induction, $t_{m}=t_{m+k}$ for all $m \geq n$. In particular, $\alpha$ is eventually periodic with period 1 hence $\alpha$ is rational.

Case $t_{n} \neq t_{n+k}$ for all $n \geq p$. First, (24) insures that (either $t_{n}=t_{n+k}+1$ or $\left.t_{n}+1=t_{n+k}\right)$ and $t_{n+1}=t_{n+1+k}$, contradicting the assumption.

Proof of Theorem 4.17. By Lemma 4.19] if $x \mapsto \alpha k^{x}$ is uniformly close to a function $f$ having integral difference ratios, then $\alpha$ is rational. Let $\alpha=m / n$ with $m, n \in \mathbb{N}$. But $\left|f(x)-(m / n) k^{x}\right|<M$ implies $\left|n f(x)-m k^{x}\right|<n M$, and $n f$ has integral difference ratios (by Proposition 3.3. closure under sum) and $n M$ is a constant, hence $x \mapsto m k^{x}$ is uniformly close to a function having integral difference ratios, contradicting Lemma 4.18

\subsection{Functions around the factorial function}

The following results make Theorem 3.1 all the more unexpected.

Proposition 4.20. If $a \in \mathbb{Z} \backslash\{0\}$ then the function $a^{x} x$ ! does not have integral difference ratios.

Proof. Let $y+1>a$ be prime and $x=2 y+1$ and observe that $x-y=$ $y+1$ divides $x$ ! hence also $a^{x} x$ ! but does not divide $a^{y} y$ ! hence does not divide $a^{x} x !-a^{y} y !$.

Due to Theorem 3.1 the following strengthening of Proposition 4.20 for the case $a=1$ fails for $a \in \mathbb{Z} \backslash\{0,1\}$. It also stresses that the one-point modification of $\lfloor e x !\rfloor$ and $\lceil e x !\rceil$ in Theorem 3.1 is no accident.

Proposition 4.21. Let $\alpha$ be a non zero real number. The functions $x \mapsto\lfloor\alpha x !\rfloor$ and $x \mapsto\lceil\alpha x !\rceil$ do not have integral difference ratios. 
Proof. We reduce to the case $\alpha>0$ since $\lfloor-r\rfloor=-\lceil r\rceil$ for $r \in \mathbb{R}$. We consider the $\lfloor\ldots\rfloor$ case, the $\lceil\ldots\rceil$ case being similar. Arguing by contradiction, assume $\lfloor\alpha \times x !\rfloor$ has integral difference ratios. Let $\theta_{a} \in[0,1[$ be such that $\alpha a !=$ $\lfloor\alpha a !\rfloor+\theta_{a}$.

First, we prove that if $: x \mapsto\lfloor\alpha x$ ! $\rfloor$ has integral difference ratios, then $\alpha$ is a rational number. Since $0 !=1 !=1$, applying the integral difference ratios property to $a \in \mathbb{N} \backslash\{0\}$ and $b=0$ and $b=1$, we see that both $a$ and $a-1$ divide $\lfloor\alpha a !\rfloor-\lfloor\alpha\rfloor$ hence (since $a$ and $a-1$ are relatively prime) $a(a-1)$ divides $\lfloor\alpha a !\rfloor-\lfloor\alpha\rfloor$. Thus, there exists $K_{a} \in \mathbb{N}$ such that

$$
\begin{aligned}
a(a-1) K_{a} & =\lfloor\alpha a !\rfloor-\lfloor\alpha 0 !\rfloor=\left(\alpha a !-\theta_{a}\right)-\left(\alpha 0 !-\theta_{0}\right) \\
& =\alpha a !+\delta_{a} \quad \text { where }\left|\delta_{a}\right| \leq 2+\alpha \\
\alpha & =\frac{K_{a}}{(a-2) !}-\frac{\delta_{a}}{a !} \\
\text { hence } \alpha & =\frac{K_{a}(a-1)}{(a-1) !}-\frac{\delta_{a}}{a !} \\
\alpha & \left.=\frac{K_{a+1}}{(a-1) !}-\frac{\delta_{a+1}}{(a+1) !} \quad \text { (replace } a \text { by } a+1 \text { in (26) }\right)
\end{aligned}
$$

Let $\operatorname{round}(\alpha, N)$ be the unique integer $x$ such that $\alpha \in\left[\frac{x}{N}-\frac{1}{2 N}, \frac{x}{N}+\frac{1}{2 N}[\right.$.

Since $\left|\delta_{a}\right|,\left|\delta_{a+1}\right|$ are bounded by $2+\alpha$ then, for $a$ large enough, (26) insures that $\operatorname{round}(\alpha,(a-2) !)=K_{a}$ whereas (27) and (28) insure that $\operatorname{round}(\alpha,(a-$ $1) !)=K_{a}(a-1)=K_{a+1}$. Thus, $K_{a}(a-1)=K_{a+1}$ hence $\frac{K_{a}}{(a-2) !}=\frac{K_{a+1}}{(a-1) !}$ is a rational constant $r$ independent of $a$ for $a$ large enough. Equations (25) and (28) insure that $|\alpha-r|<(2+\alpha) /(a+1)$ ! for all $a$ big enough; hence $|\alpha-r|$ is arbitrarily small and thus $\alpha=r$ is a rational number.

We can now get the wanted contradiction. Let $\alpha=p / q$ where $p, q$ are relatively prime. Let $a$ be such that $a-q$ is prime and $a-q>p q$ !. Since $a>q$, we have $\alpha a !=p(a !) / q \in \mathbb{N}$ hence $\lfloor\alpha a !\rfloor=\alpha a !$ and $a-q$ divides $\lfloor\alpha a !\rfloor$.

Also, $\alpha q !=p(q-1) ! \in \mathbb{N}$ hence $\lfloor\alpha q !\rfloor=\alpha q !=p(q-1) !$. Since $a-q$ is prime and $a-q>p q$ !, it cannot divide $\lfloor\alpha q !\rfloor$. Thus, $a-q$ cannot divide $\lfloor\alpha a !\rfloor-\lfloor\alpha q !\rfloor$, contradicting the integral difference ratios assumption.

Theorem 4.22 shows that the uniform closeness analog of Corollary 3.16 fails.

Theorem 4.22. For $a \in \mathbb{R} \backslash\{0,1\}$, let $X_{a}$ be the set of real numbers $\alpha$ such that the map $x \mapsto \alpha a^{x} x$ ! from $\mathbb{N}$ to $\mathbb{R}$ is uniformly close to some function having integral difference ratios.

1. For every $a \in \mathbb{R} \backslash\{0\}$, the set $X_{a}$ has Lebesgue measure zero.

2. If $a \in \mathbb{Z} \backslash\{0\}$, the set $X_{a}$ contains $e^{1 / a} \mathbb{Z}$ but misses every non null rational number.

Proof. 1. Applied with $\lambda_{n}=a^{n} n$ !, Theorem 4.9 insures that $X_{a}$ has measure zero.

2. Inclusion $X_{a} \supseteq e^{1 / a} \mathbb{N}$, for $a \in \mathbb{Z} \backslash\{0\}$, is a consequence of Corollary 3.5.

By way of contradiction, assume some non null rational number $\alpha$ is in $X_{a}$. Let $\alpha=p / q$ where $p \in \mathbb{Z}$ and $q \in \mathbb{N} \backslash\{0,1\}$. Let $f: \mathbb{N} \rightarrow \mathbb{Z}$ and $M \in \mathbb{N}$ be such that $f$ has integral difference ratios and $\left|f(x)-(p / q) a^{x} x !\right|<M$ for all $x \in \mathbb{N}$. Thus,

$$
q f(x)=p a^{x} x !+\varepsilon_{x} q M \quad \text { with }\left|\varepsilon_{x}\right|<1 .
$$


Choose $y=2 q M$ and $x=p a^{y} y !+y+1$. Every divisor of $q$ divides $y$ and $x-y-1$ hence does not divide $x-y$. Thus, $q$ and $x-y$ are coprime. Since $q, x-y<x$, we see that $q(x-y)$ divides $x$ ! hence also $p a^{x} x$ !. Since $q(x-y)$ divides $q(f(x)-f(y))$, we see that $q(x-y)$ divides

$$
\begin{aligned}
p a^{x} x !-q(f(x)-f(y)) & =p a^{x} x !-\left(p a^{x} x !+\varepsilon_{x} q M\right)+\left(p a^{y} y !+\varepsilon_{y} q M\right) \\
& =p a^{y} y !+\ell_{x, y}
\end{aligned}
$$

where $\left|\varepsilon_{x}\right|,\left|\varepsilon_{1}\right|<1$ and $\left|\ell_{x, y}\right|<2 q M=y$, whence $p a^{y} y !+\ell_{x, y}<p a^{y} y !+y$. Then $q(x-y)=q\left(p a^{y} y !+1\right)>2 p a^{y} y$ ! cannot divide $p a^{y} y !+\ell_{x, y}<p a^{y} y !+y<$ $2 p a^{y} y$ !. Contradiction.

How complex are the real numbers in the set $X_{a}$ of Theorem 4.22? First, we recall the notions of irrationality measure and Liouville numbers.

Definition 4.23. 1. The irrationality exponent of a real number $\alpha$ is the supremum of all $\mu \in \mathbb{R}^{+}$such that the approximation $|\alpha-(p / q)|<1 / q^{\mu}$ holds for infinitely many rational numbers $p / q$.

2. A real number is Liouville if its irrationality exponent is infinite.

Proposition 4.24. 1. Rational numbers have irrationality exponent 1.

2. Irrational numbers have irrationality exponent at least 2.

3. (Roth, 1955) All irrational algebraic numbers have irrationality exponent 2.

4. (Khinchin, 1924, cf. [21] p.17) Almost all real numbers have irrationality exponent 2 .

For $a \in \mathbb{Z} \backslash\{0\}$, the sole numbers known to be in the set $X_{a}$ of Theorem 4.22 are those in the set $e^{1 / a} \mathbb{Z}$. It turns out that all have irrationality exponent equal to 2 .

Proposition 4.25. All numbers $s e^{1 / a}, a, s \in \mathbb{Z} \backslash\{0\}$, have irrationality exponent 2. In particular, though they are transcendental, they are not Liouville.

Proof. It obviously suffices to consider $s=1$. First, we consider the case $a \in$ $\mathbb{N} \backslash\{0\}$.

The continued fraction expansion $\left[a_{0} ; a_{1}, a_{2}, \ldots\right]$ of $e^{1 / a}$ was computed by Euler (cf. 25] page 10):

$$
\begin{aligned}
& e=[2,1,2,1,1,4,1,1,6,1,1, \ldots]=[2 ; \overline{1,2 m, 1}]_{m \in \mathbb{N}} \\
& \text { for } a \geq 2, \quad e^{1 / a}=[1 ; a, 1,1,3 a, 1,1,5 a, \ldots] \quad=[\overline{1,(2 m+1) a, 1}]_{m \in \mathbb{N}}
\end{aligned}
$$

Let $p_{n} / q_{n}$ be its $n$-th convergent. As a general result for all irrational numbers (cf. [10] Theorems 6, 12 and $9 \& 13$ ), we have

$$
\begin{aligned}
\frac{q_{n+1}}{q_{n}} & =\left[a_{n+1} ; a_{n}, \ldots, a_{1}\right]<a_{n+1}+1 \\
q_{n} & \leq 2^{(n-1 / 2)} \\
\frac{1}{q_{n}\left(q_{n}+q_{n+1}\right)} & <\left|e^{1 / a}-\frac{p_{n}}{q_{n}}\right|<\frac{1}{q_{n} q_{n+1}}
\end{aligned}
$$

The above Euler formulas show that, the continued fraction expansion $e^{1 / a}$ is such that, $a_{n+1} \leq n-1$ for $n \geq 3$. Thanks to (30), we get $q_{n+1} \leq n q_{n}$. Using 
(31), for any $\varepsilon>0$, this yields $q_{n}\left(q_{n}+q_{n+1}\right) \leq(n+1) q_{n}^{2} \leq q_{n}^{2+\varepsilon}$ for $n$ large enough. Reporting in (32), we finally have

$$
\frac{1}{q_{n}^{2+\varepsilon}}<\left|e^{1 / a}-\frac{p_{n}}{q_{n}}\right|<\frac{1}{q_{n}^{2}}
$$

which proves that the irrationality exponent of $e^{1 / a}$ is 2 . The argument also applies to $e^{-1 / a}=1 / e^{1 / a}$ since its continued fraction expansion is $\left[0 ; a_{0}, a_{1}, a_{2}, \ldots\right]$.

Open problem. Does the set $X_{a}$ of Theorem 4.22 contain numbers with irrationality measure other than 2 ?

We can only prove that it misses a subfamily of Liouville numbers.

Definition 4.26. Let $\theta: \mathbb{N} \rightarrow \mathbb{N}$ be such that, for all $q \in \mathbb{N}$, the map $n \mapsto \theta(q, n)$ goes to infinity. A real number $\alpha$ is $\theta$-Liouville if, for all $n \in \mathbb{N}$, there exists a rational number $p / q$ such that

$$
\left|\alpha-\frac{p}{q}\right|<\frac{1}{\theta(q, n)} .
$$

Theorem 4.27. Let exp $3: \mathbb{N} \rightarrow \mathbb{N}$ and $\theta: \mathbb{N} \rightarrow \mathbb{N}$ be such that $\exp 3(x)=x^{x^{x}}$ and $\theta(q, n)=\exp 3(q n)$.

1. All $\theta$-Liouville numbers are Liouville numbers.

2. For $a \in \mathbb{Z} \backslash\{0,1\}$, the set $X_{a}$ misses all $\theta$-Liouville numbers.

Remark 4.28. Recall (cf. [10] Theorem 22, or [21] Theorem 1 p.12) that, for any $\theta$, there exist $\theta$-Liouville real numbers. For the above $\theta$, an example is $\sum_{n \in \mathbb{N}} \frac{1}{a_{n}}$ where $a_{0}=2$ and $a_{n+1}=2^{a_{n}+\exp 3\left(n a_{n}\right)}$.

Proof of Theorem 4.22. 1. Obvious since $\theta(q, n) \geq q^{n}$.

2. Let $\alpha$ be $\theta$-Liouville. With no loss of generality, we can assume $\alpha>0$. Let $C \in \mathbb{N}$ be such that $\alpha<C$. Then

$$
\forall n \in \mathbb{N} \quad \exists p \in \mathbb{Z} \quad \exists q \in \mathbb{N} \backslash\{0\} \quad\left(\left|\alpha-\frac{p}{q}\right|<\frac{1}{q \exp 3(n q)} \text { and } p<A q\right) .
$$

By way of contradiction, suppose $\alpha$ is in $X_{a}$ and let $f: \mathbb{N} \rightarrow \mathbb{Z}$ and $M \in \mathbb{N}$, $M \geq 1$, be such that $f$ has integral difference ratios and $\left|f(x)-\alpha a^{x} x !\right|<M$ for all $x \in \mathbb{N}$. We argue in a way similar to that in the proof of Theorem 4.22,

Choose $y=5 q M+C$ and $x=p a^{y} y !+y+1$. Observe that, for $n$ large enough, we have

$$
a^{x} x !+a^{y} y ! \leq q M \exp 3(n q)
$$

In fact, by Stirling formula, there exist $B, C$ (depending only on $C$ and $M$ ) such that, for $n>B$,

$$
x \leq(A q)^{A q} \quad, \quad a^{x} x !+a^{y} y ! \leq(B q)^{(B q)^{B q}}<q M \exp 3(n q)
$$

Choose such an $n \in \mathbb{N}$ and let $p, q$ be as in (34). Then, for all $z \in \mathbb{N}$,

$$
q f(z)=q \alpha a^{z} z !+\varepsilon q M=p a^{z} z !+\delta \frac{a^{z} z !}{\exp 3(n q)}+\varepsilon q M
$$


where $|\varepsilon|,|\delta|<1$. Arguing as for the case $\alpha$ rational, we see that $q(x-y)$ divides $p a^{x} x !-q(f(x)-f(y))$. Thus, to get a contradiction, it suffices to show

$$
0<p a^{x} x !-q(f(x)-f(y))<x-1 .
$$

Now, (36) insures that

$$
p a^{x} x !-q(f(x)-f(y))=p a^{y} y !+\ell_{x, y}+\Delta \frac{a^{x} x !+a^{y} y !}{\exp 3(n q)}
$$

Using (35) and inequalities $\left|\ell_{x, y}\right|<2 q M$, and $|\Delta|<1$, we see that

$$
\left|\ell_{x, y}+\Delta \frac{a^{x} x !+a^{y} y !}{\exp 3(n q)}\right| \leq y
$$

hence

$$
0<p a^{y} y !-y<p a^{y} y !+\ell_{x, y}+\Delta \frac{a^{x} x !+a^{y} y !}{\exp 3(n q)}<p a^{y} y !+y=x-1 .
$$

This gives the wanted inequality and concludes the proof.

\section{References}

[1] G. Boole, A treatise on the calculus of finite differences, London, 1872. Reedition Cosimo Classics, 2007.

[2] P. CÉgielski and S. Grigorieff and I. Guessarian, On Lattices of Regular Sets of Natural Integers Closed under Decrementation, Submitted, 2013. Preprint version on arXiv, 2013.

[3] P. Dusart, Estimates of some functions over primes without Riemann hypothesis, unpublished. Preprint version on arXiv, 2010.

[4] P.L. Chebyshev, Mémoire sur les nombres premiers, Journal de Mathématiques Pures et Appliquées (1), 17:366-390, 1852.

[5] B. FARHI, Nontrivial lower bounds for the least common multiple of some finite sequences of integers, Journal of Number Theory, 125:393411, 2007.

[6] B. Farhi and D. Kane, New Results on the Least Common Multiple of Consecutive Integers, Proceedings of the AMS, 137(6):1933-1939, 2009.

[7] R. Graham and D. Knuth and O. Patashnik, Concrete Mathematics: A Foundation for Computer Science, Addison-Wesley, 1994.

[8] D. Hanson, On the product of the primes, Canadian Mathematical Bulletin, 15(1):33-37, 1972.

[9] S. Hong, G. QIAn and Q. Tan, The least common multiple of a sequence of products of linear polynomials, Acta Mathematica Hungarica, 135(1-2):160-167, 2011.

[10] A. Ya. Khinchin, Continued fractions, University of Chicago Press, 1964.

[11] L. Kuipers AND H. NiederReiter, Uniform distribution of sequences, Dover, 1974.

[12] D. Kwon, A devils staircase from rotations and irrationality measures for Liouville numbers, Mathematical Proceedings of the Cambridge Philosophical Society, 145:739-756, 2008.

[13] Martin E. Muldoon, Generalized hyperbolic functions, circulant matrices and functional equations, Linear Algebra and its Applications, 406:272284, 2005. Preprint version on arXiv. 
[14] M. NAIR, On Chebyshev-type inequalities for primes, The American Mathematical Monthly, 89(2):126-129, 1982.

[15] I. Newton, Philosophice Naturalis Principia Mathematica, London, 1687 (revised 3rd edition, 1726).

[16] I. Niven, Irrational Numbers, Carus Mathematical Monograph 1956.

[17] J.-É. PIN AND P.V. Silva, On profinite uniform structures defined by varieties of finite monoids, International Journal of Algebra and Computation, 21:295-314, 2011.

[18] J.-É. Pin AND P.V. Silva, On uniformly continuous functions for some profinite topologies, in preparation.

[19] Guoyou Qian and Shaofang Hong, Asymptotic behavior of the least common multiple of consecutive arithmetic progression terms, Archiv der Mathematik, 100(4):337-345, 2013.

[20] J.B. Rosser And L. Schoenfeld, Sharper Bounds for the Chebyshev Functions $\theta(x)$ and $\psi(x)$, Mathematics of computation, 29(129):243-269, 1975.

[21] A. B. ShidLovskiI, Transcendental numbers, De Gruyter, 1989.

[22] E. Schmidt, Über die Anzahl der Primzahlen unter gegebener Grenze, Mathematische Annalen, 57(2):195-204, 1903.

[23] L. Schoenfeld, Sharper Bounds for the Chebyshev Functions $\theta(x)$ and $\psi(x)$. II, Mathematics of computation, 30(134):337-360, 1976.

[24] A. Ungar, Generalized hyperbolic functions, The American Mathematical Monthly, 89(9):688-691, 1982.

[25] M. WAldSChmidt, An introduction to irrationality and transcendence methods, Lecture notes of Arizona Winter School "Special functions and transcendence", The University of Arizona, 2008. Cf. http://www.math.jussieu.fr//miw/articles/pdf/AWSLecture1.pdf

[26] S.L. YANG AND Z.K. LIU, Explicit inverse of the Pascal matrix plus one, International Journal of Mathematics and Mathematical Sciences, $1-7,2006$. 